\title{
A Holistic Perspective on the Dynamics of G035.39-00.33 : The Interplay between Gas and Magnetic Fields
}

Liu, Tie

2018-06-01

Liu , T , Li , P S , Juvela , M , Kim , K-T , Evans , N J , Di Francesco , J , Liu , S-Y , Yuan , J , Tatematsu , K, Zhang , Q , Ward-Thompson, D , Fuller, G, Goldsmith , P F , Koch , P M , Sanhueza , P , Ristorcelli , I , Kang , S, Chen , H-R , Hirano , N, Wu , Y, Sokolov , V , Lee , C W , White , G J , Wang, K, Eden, D , Li , D , Thompson, M , Pattle , K M, Soam , A, Nasedkin , E , Kim , J , Kim , G , Lai , S-P , Park, G, Qiu , K, Zhang , C-P , Alina , D , Eswaraiah, C, Falgarone , E , Fich, M , Greaves , J, Gu, Q -L , Kwon , W , Li , H , Malinen , J , Montier , L , Parsons , H , Qin , S-L , Rawlings , M G \& Tang , Y -W 2018 , ' A Holistic Perspective on the Dynamics of G035.39-00.33 : The Interplay between Gas and Magnetic Fields ' , Astrophysical Journal , vol. 859 , no. 2 , 151 . https://doi.org/10.3847/1538-4357/aac025

http://hdl.handle.net/10138/236644

https://doi.org/10.3847/1538-4357/aac025

unspecified

publishedVersion

Downloaded from Helda, University of Helsinki institutional repository.

This is an electronic reprint of the original article.

This reprint may differ from the original in pagination and typographic detail.

Please cite the original version. 


\title{
A Holistic Perspective on the Dynamics of G035.39-00.33: The Interplay between Gas and Magnetic Fields
}

Tie Liu ${ }^{1,2}$ (D), Pak Shing Li $^{3}$ (D), Mika Juvela ${ }^{4}$, Kee-Tae Kim ${ }^{1}$ (D), Neal J. Evans II $^{1,5}$, James Di Francesco ${ }^{6,7}$, Sheng-Yuan Liu ${ }^{8}$, Jinghua Yuan $^{9}$ (D), Ken'ichi Tatematsu ${ }^{10}$ (D), Qizhou Zhang ${ }^{11}$ (D), Derek Ward-Thompson ${ }^{12}$ (D), Gary Fuller ${ }^{13}$, Paul F. Goldsmith ${ }^{14}$ (D),

P. M. Koch ${ }^{8}$ (D) Patricio Sanhueza ${ }^{10}$ (D), I. Ristorcelli ${ }^{15}$, Sung-ju Kang ${ }^{1}$ (D), Huei-Ru Chen ${ }^{16}$ (D), N. Hirano ${ }^{8}$, Yuefang Wu ${ }^{17}$ (D),

Vlas Sokolov $^{18}$ (D), Chang Won Lee ${ }^{1,19}$, Glenn J. White ${ }^{20,21}$ (D), Ke Wang ${ }^{22}$ (D), David Eden ${ }^{23}$, Di Li ${ }^{9,24}$, Mark Thompson ${ }^{25}$,

Kate M Pattle $^{16}$ (D) , Archana Soam ${ }^{1}$ (D), Evert Nasedkin ${ }^{26}$, Jongsoo Kim ${ }^{1}$, Gwanjeong Kim ${ }^{10}$, Shih-Ping Lai ${ }^{16}$ (iD), Geumsook Park ${ }^{1}$ (D),

Keping Qiu ${ }^{27}$ (D), Chuan-Peng Zhang ${ }^{9}$ (D), Dana Alina ${ }^{28}$, Chakali Eswaraiah ${ }^{16}$ (D), Edith Falgarone ${ }^{29}$, Michel Fich ${ }^{26}$,

Jane Greaves $^{30}$ (D), Q.-L. Gu ${ }^{31}$, Woojin Kwon ${ }^{1,19}$ (D), Hua-bai Li ${ }^{31}$ (D), Johanna Malinen ${ }^{32}$, Ludovic Montier ${ }^{15}$, Harriet Parsons ${ }^{2}$ (D), Sheng-Li Qin ${ }^{33}$, Mark G. Rawlings ${ }^{2}$, Zhi-Yuan Ren ${ }^{9}$, Mengyao Tang ${ }^{33}$ (D), Y.-W. Tang ${ }^{8}$, L. V. Toth ${ }^{34}$ (D), Jiawei Wang ${ }^{16}$, Jan Wouterloot ${ }^{2}$ (D) H.-W. Yi ${ }^{35}$, and H.-W. Zhang ${ }^{17}$

${ }^{1}$ Korea Astronomy and Space Science Institute, 776 Daedeokdaero, Yuseong-gu, Daejeon 34055, Republic of Korea; liutiepku@gmail.com ${ }^{2}$ East Asian Observatory, 660 N. A'ohoku Place, Hilo, HI 96720, USA

${ }^{3}$ Astronomy Department, University of California, Berkeley, CA 94720, USA

${ }^{4}$ Department of Physics, P.O. Box 64, FI-00014, University of Helsinki, Finland

${ }^{5}$ Department of Astronomy, University of Texas at Austin, 2515 Speedway, Stop C1400, Austin, TX 78712-1205, USA

${ }^{6}$ NRC Herzberg Astronomy and Astrophysics, 5071 West Saanich Rd., Victoria, BC V9E 2E7, Canada

${ }^{7}$ Department of Physics and Astronomy, University of Victoria, Victoria, BC V8P 5C2, Canada

${ }^{8}$ Institute of Astronomy and Astrophysics, Academia Sinica. 11F of Astronomy-Mathematics Building, AS/NTU No.1, Sec. 4, Roosevelt Rd, Taipei 10617, Taiwan

${ }^{9}$ National Astronomical Observatories, Chinese Academy of Sciences, Beijing, 100012, People's Republic of China

${ }^{10}$ National Astronomical Observatory of Japan, National Institutes of Natural Sciences, 2-21-1 Osawa, Mitaka, Tokyo 181-8588, Japan

${ }^{11}$ Harvard-Smithsonian Center for Astrophysics, 60 Garden Street, Cambridge, MA 02138, USA

12 Jeremiah Horrocks Institute for Mathematics, Physics \& Astronomy, University of Central Lancashire, Preston PR1 2HE, UK

${ }^{13}$ UK ALMA Regional Centre Node, Jodrell Bank Centre for Astrophysics, School of Physics and Astronomy, The University of Manchester, Oxford Road, Manchester M13 9PL, UK

${ }^{14}$ Jet Propulsion Laboratory, California Institute of Technology, 4800 Oak Grove Drive, Pasadena, CA 91109, USA

${ }^{15}$ IRAP, Université de Toulouse, CNRS, UPS, CNES, Toulouse, France

${ }^{16}$ Institute of Astronomy and Department of Physics, National Tsing Hua University, Hsinchu, Taiwan

${ }_{17}$ Department of Astronomy, Peking University, 100871, Beijing, People's Republic of China

${ }_{18}^{18}$ Max Planck Institute for Extraterrestrial Physics, Gießenbachstraßse 1, D-85748, Garching bei München, Germany

${ }^{19}$ Korea University of Science and Technology, 217 Gajeong-ro, Yuseong-gu, Daejeon 34113, Republic of Korea

${ }^{20}$ Department of Physics and Astronomy, The Open University, Walton Hall, Milton Keynes, MK7 6AA, UK

${ }^{21}$ RAL Space, STFC Rutherford Appleton Laboratory, Chilton, Didcot, Oxfordshire, OX11 0QX, UK

${ }^{22}$ European Southern Observatory, Karl-Schwarzschild-Str. 2, D-85748 Garching bei München, Germany

${ }^{23}$ Astrophysics Research Institute, Liverpool John Moores University, IC2, Liverpool Science Park, 146 Brownlow Hill, Liverpool L3 5RF, UK

${ }^{24}$ Key Laboratory of Radio Astronomy, Chinese Academy of Science, Nanjing 210008, People's Republic of China

${ }^{25}$ Centre for Astrophysics Research, School of Physics Astronomy \& Mathematics, University of Hertfordshire, College Lane, Hatfield, AL10 9AB, UK

${ }^{26}$ Department of Physics and Astronomy, University of Waterloo, Waterloo, ON N2L 3G1, Canada

${ }^{27}$ School of Astronomy and Space Science, Nanjing University, Nanjing 210023, People's Republic of China

${ }^{28}$ Department of Physics, School of Science and Technology, Nazarbayev University, Astana 010000, Kazakhstan

${ }^{29}$ LERMA, Observatoire de Paris, PSL Research University, CNRS, Sorbonne Universités, UPMC Univ. Paris 06, Ecole normale supérieure, F-75005 Paris, France

${ }^{30}$ School of Physics and Astronomy, Cardiff University, Cardiff CF24 3AA, UK

${ }^{31}$ Department of Physics, The Chinese University of Hong Kong, Shatin, New Territory, Hong Kong, People's Republic of China

32 Institute of Physics I, University of Cologne, Zülpicher Str. 77, D-50937, Cologne, Germany

${ }^{33}$ Department of Astronomy, Yunnan University, and Key Laboratory of Astroparticle Physics of Yunnan Province, Kunming, 650091, People's Republic of China

${ }^{34}$ Eötvös Loránd University, Department of Astronomy, Pázmány Péter sétány 1/A, H-1117, Budapest, Hungary

${ }^{35}$ School of Space Research, Kyung Hee University, Yongin-Si, Gyeonggi-Do 17104, Republic of Korea

Received 2018 January 25; revised 2018 April 23; accepted 2018 April 23; published 2018 June 4

\begin{abstract}
Magnetic field plays a crucial role in shaping molecular clouds and regulating star formation, yet the complete information on the magnetic field is not well constrained owing to the limitations in observations. We study the magnetic field in the massive infrared dark cloud G035.39-00.33 from dust continuum polarization observations at $850 \mu \mathrm{m}$ with SCUBA-2/POL-2 at JCMT for the first time. The magnetic field tends to be perpendicular to the densest part of the main filament $\left(F_{\mathrm{M}}\right)$, whereas it has a less defined relative orientation in the rest of the structure, where it tends to be parallel to some diffuse regions. A mean plane-of-the-sky magnetic field strength of $\sim 50 \mu \mathrm{G}$ for $F_{\mathrm{M}}$ is obtained using the Davis-Chandrasekhar-Fermi method. Based on ${ }^{13} \mathrm{CO}(1-0)$ line observations, we suggest a formation scenario of $F_{\mathrm{M}}$ due to large-scale $(\sim 10 \mathrm{pc})$ cloud-cloud collision. Using additional $\mathrm{NH}_{3}$ line data, we estimate that $F_{\mathrm{M}}$ will be gravitationally unstable if it is only supported by thermal pressure and turbulence. The northern part of $F_{\mathrm{M}}$, however, can be stabilized by a modest additional support from the local magnetic field. The middle and southern parts of $F_{\mathrm{M}}$ are likely unstable even if the magnetic field support is taken into account. We claim that the clumps in $F_{\mathrm{M}}$ may be supported by turbulence and magnetic fields against gravitational collapse. Finally, we identified for the first time a massive $\left(\sim 200 M_{\odot}\right)$, collapsing
\end{abstract}


starless clump candidate, “c8," in G035.39-00.33. The magnetic field surrounding "c8" is likely pinched, hinting at an accretion flow along the filament.

Key words: ISM: clouds - ISM: magnetic fields - stars: formation

\section{Introduction}

The densest parts of massive molecular dark clouds are filamentary in form, with lengths ranging from several parsecs to more than $10 \mathrm{pc}$ and with a width of a few tenths of a parsec (André et al. 2014; Wang et al. 2016). One of the most striking results from Herschel observations in the Gould Belt clouds is the finding of an apparent characteristic width $(\sim 0.1 \mathrm{pc})$ of filamentary substructures (André et al. 2014). The origin of such a characteristic width is not well understood. Projection effects or artifacts in the data analysis may also affect this result (Panopoulou et al. 2017). However, numerical simulations modeling the interplay between turbulence, strong magnetic field, and gravitationally driven ambipolar diffusion are indeed able to reproduce filamentary structures with widths peaked at $0.1 \mathrm{pc}$ over several orders of magnitude in column density (e.g., Auddy et al. 2016; Federrath 2016). Therefore, it is crucial to investigate the interplay between turbulence, magnetic field, and gravity in filamentary clouds to understand their properties.

Statistical analysis of observed magnetic fields in the nearby Taurus, Musca, Ophiuchus, Chameleon, and Vela C molecular clouds, as well as many infrared dark clouds (IRDCs), has revealed that the local magnetic fields tend to be perpendicular to the densest filaments, whereas the fields tend to be parallel in the lower-density peripheries of those filaments (Chapman et al. 2011; Li et al. 2015a; Cox et al. 2016; Malinen et al. 2016; Planck Collaboration et al. 2016a, 2016b; Santos et al. 2016; Alina et al. 2017; Soler et al. 2017; Ward-Thompson et al. 2017; Tang et al. 2018b).

Recent state-of-the-art large-scale ideal magnetohydrodynamic (MHD) simulations of the formation and structure of filamentary dark clouds suggest a complicated evolutionary process involving the interaction and fragmentation of dense, velocity-coherent fibers into chains of cores (e.g., Klassen et al. 2017; Li et al. 2017). In the simulation of Li et al. (2017), the global magnetic field is roughly perpendicular to the long axis of the main filamentary cloud. Velocity-coherent fibers are identified inside the filamentary cloud and appear to be intertwined along the main filamentary cloud. These results are similar to the structures identified in L1495/B213 (see Hacar et al. 2013, 2016). In three-dimensional MHD simulations of cluster-forming turbulent molecular cloud clumps, Klassen et al. (2017) find that magnetic fields are oriented more parallel to the major axis of the subvirial clouds and more perpendicular in the denser and marginally bound clouds. Observationally, similar results are found by Koch et al. (2014) where the local angle $(|\delta|)$ between an intensity gradient and a magnetic field orientation shows a possible bimodal distribution and clearly separates subcritical from supercritical cores, based on 50 sources observed with the Submillimeter Array (SMA) and the the Caltech Submillimeter Observatory (CSO).

Both numerical simulations (Li et al. 2015b, 2017; Klassen et al. 2017; Soler \& Hennebelle 2017) and polarization observations (Li et al. 2009, 2015a; Chapman et al. 2011; Koch et al. 2012; Girart et al. 2013; Koch et al. 2014; Qiu et al. 2013, 2014; Zhang et al. 2014; Pillai et al. 2015; Cox et al. 2016; Pattle et al. 2017; Ward-Thompson et al. 2017) have found that the interstellar magnetic field is dynamically important to the formation of dense cores in filamentary clouds. It is, however, still unclear how important the magnetic field is in the formation of dense cores in filaments relative to the turbulence and gravity.

Optical or near-infrared absorption polarimetry that can trace the plane-of-the-sky (POS) projections of magnetic field orientations has been limited to low-density, diffuse cloud material. Polarized submillimeter thermal dust emission, however, can trace magnetic fields in dense regions of clouds. Planck submillimeter polarimetry, while extensive, is limited to the study of distant clouds (e.g., IRDCs) owing to the low angular resolution $\left(\sim 5^{\prime}\right.$ or $\sim 1.5 \mathrm{pc}$ at $1 \mathrm{kpc}$ distance; Planck Collaboration et al. 2016a, 2016b; Alina et al. 2017). High angular resolution observations of polarized submillimeter thermal dust emission toward filamentary clouds are much better at tracing the cores but are still very rare. Such observations, specifically of quiescent filamentary clouds that are not greatly affected by the star-forming activities, are needed to explore the roles of magnetic field in dense core formation in filamentary clouds. One example of a massive but quiescent filamentary cloud is IRDC G035.39-00.33 (hereafter denoted as G035.39).

Located at a distance of $2.9 \mathrm{kpc}$ (Simon et al. 2006), G035.39 is an IRDC with a total mass of $\sim 16,700 M_{\odot}$ (Kainulainen \& Tan 2013). G035.39 contains massive, dense clumps as revealed by dense molecular line observations (Henshaw et al. 2013, 2014, 2017; Jiménez-Serra et al. 2014; Zhang et al. 2017). Kinematically identified substructures and resolved narrow $(0.028 \pm 0.005 \mathrm{pc})$ fibers have been identified in G035.39 (Henshaw et al. 2017), indicating the existence of interacting velocity-coherent fibers similar to those discovered in L1495/ B213. High CO depletion factors $\left(f_{\mathrm{D}} \sim 5-10\right.$; Jiménez-Serra et al. 2014) and a high deuterium fractionation $\left(\mathrm{D}_{\mathrm{N}_{2} \mathrm{H}^{+}}\right)$of $\mathrm{N}_{2} \mathrm{H}^{+}$ (mean $\mathrm{D}_{\mathrm{N}_{2} \mathrm{H}^{+}}=0.04 \pm 0.01$; Barnes et al. 2016) in the dense cores of G035.39 indicate that G035.39 is chemically evolved but has been relatively unaffected by the ongoing star-forming activities. Indeed, the dense cores in this filament are either starless or associated with very low luminosity "Class 0"-like IR-quiet protostars (Nguyen Luong et al. 2011).

G035.39 is also known as a Planck Galactic Cold Clump (PGCC), PGCC G35.49-0.31 (Planck Collaboration et al. 2016c). PGCCs are ideal targets for investigating the initial conditions of star formation and for studying the properties of filamentary clouds (Juvela et al. 2010, 2012; Planck Collaboration et al. 2011a, 2011b, 2016c; Liu et al. 2012, 2013c, 2015; Wu et al. 2012; Meng et al. 2013; Montillaud et al. 2015; RiveraIngraham et al. 2016, 2017; Yuan et al. 2016; Zhang et al. 2016; Tatematsu et al. 2017). G035.39 has been observed as part of the JCMT legacy survey program "SCUBA-2 Continuum Observations of Pre-protostellar Evolution (SCOPE)," which targets $\sim 1000$ PGCCs in $850 \mu \mathrm{m}$ continuum and suitable for the investigation of the initial conditions of star formation in widely different Galactic environments (Liu et al. 2016c, 2018; Juvela et al. 2018a; Kim et al. 2017; Tang et al. 2018a; Yi et al. 2018; Zhang et al. 2018). The "SCOPE" survey has provided us thousands of dense clumps (D. Eden et al. 2018, in preparation; Liu et al. 2018) for these studies. 
The magnetic field surrounding G035.39 may not be affected by star-forming activities (like outflows); therefore, G035.39 is an ideal target for polarization observations of initial conditions for the formation of IRDCs. To this end, we conducted a number of linear polarization observations of the dust continuum emission at $850 \mu \mathrm{m}$ with the new POL-2 polarimeter, operating in conjunction with Submillimeter Common User Bolometer Array 2 (SCUBA-2) at the James Clerk Maxwell Telescope (JCMT). The SCUBA-2/POL-2 observations of G035.39 serve as a pilot study of magnetic fields in "SCOPE" objects. The kinematics of the structures in G035.39 is also investigated thoroughly from molecular line observations.

Our paper is organized as follows. In Section 2, we discuss our observations using SCUBA-2/POL-2 $850 \mu \mathrm{m}$ polarization continuum, together with other continuum data used to study the spectral energy distribution of G035.39. We also present our molecular line observations. In Section 3, we present the results from these observational data, and in Section 4 we discuss the implication of the data relevant to filamentary cloud formation induced by the cloud-cloud collision (Section 4.1), the origin of magnetic field geometry (Section 4.2), the gravitational stability of the filaments (Section 4.3), and the physical properties of clumps inside G035.39 (Section 4.4). We summarize our findings in Section 5.

\section{Observations}

\subsection{Polarized $850 \mu \mathrm{m}$ Continuum Data}

The POS magnetic field is traced by polarized $850 \mu \mathrm{m}$ continuum data obtained with the SCUBA-2/POL-2 instrument at the JCMT. The SCUBA-2/POL-2 observations of G035.39 (project code: M17BP050; PI: Tie Liu) were conducted from 2017 June to 2017 November using a version of the SCUBA-2 DAISY mapping mode (Holland et al. 2013) optimized for POL-2 observations (POL-2 DAISY mapping mode; Friberg et al. 2016). ${ }^{37}$ In total, 70 scans were conducted. The beam size of the JCMT at $850 \mu \mathrm{m}$ is 14 ." 1 . The POL-2 DAISY scan pattern uses a scan speed of $8^{\prime \prime} \mathrm{s}^{-1}$ (compared to $155^{\prime \prime} \mathrm{s}^{-1}$ for a SCUBA-2 DAISY scan pattern) and a fully sampled circular region with a diameter of $12^{\prime}$, with a waveplate rotation speed of $2 \mathrm{~Hz}$ (Ward-Thompson et al. 2017). The full description of the SCUBA-2/POL-2 instrument and the POL-2 observational mode can be found in Friberg et al. (2016) and Ward-Thompson et al. (2017). Since only the central $3^{\prime}$ diameter region has an approximately uniform coverage in the POL-2 DAISY observations, we obtained two adjacent maps to cover G035.39. The central pointings of the two maps are R.A.(J2000) $=18: 57: 07$, decl. $(\mathrm{J} 2000)=+02: 11: 30$ and R.A. $(\mathrm{J} 2000)=18: 57: 10$, decl. $(\mathrm{J} 2000)=+02: 08: 00$.

Data reduction is performed using a python script called pol2map written within the STARLINK/SMURF package (Chapin et al. 2013), which is specific for submillimeter data reduction (much of it specific to the JCMT). The default pixel size in SCUBA-2/POL-2 observations is $4^{\prime \prime}$, but the final data are gridded to $8^{\prime \prime}$ pixels in pol2map to improve sensitivity. The Stokes $Q, U$, and $I$ data are all reduced with a filtering-out scale of $200^{\prime \prime}$. The output polarization percentage values are debiased using the mean of their $\mathrm{Q}$ and $\mathrm{U}$ variances to remove statistical biasing in regions of low signal-to-noise (Kwon et al. 2018; Soam et al. 2018). The details of data reduction with pol2map

\footnotetext{
37 http://www.eaobservatory.org/jcmt/instrumentation/continuum/scuba-2/ pol-2/
}

can be found in Kwon et al. (2018) and Soam et al. (2018). The final co-added maps have an rms noise of $\sim 1.5 \mathrm{mJy} \mathrm{beam}^{-1}$. The polarization angle $\theta$ is measured as $\theta=0.5 \arctan (U / Q)$. The angle increases from north toward east, following the IAU convention. Throughout this paper, the polarization orientations obtained are rotated by $90^{\circ}$ to show the magnetic field orientation projected on the POS.

\subsection{Continuum Data}

We use SCUBA-2 Stokes $I 450$ and $850 \mu \mathrm{m}$ continuum data obtained from the legacy survey program "SCOPE" (Liu et al. 2018) and Herschel archival data from the Hi-GAL project (Molinari et al. 2010) to construct the pixel-by-pixel SEDs of the G035.39 field.

The SCUBA-2 observations were conducted on 2016 April 13 under better weather conditions than SCUBA-2/POL-2 observations in 2017. Therefore, the $450 \mu \mathrm{m}$ data were also obtained. The beam sizes at 450 and $850 \mu \mathrm{m}$ are 7 !" 9 and 14 ". 1 , respectively. The pixel sizes are $2^{\prime \prime}$ and $4^{\prime \prime}$ at 450 and $850 \mu \mathrm{m}$, respectively. The rms levels at 450 and $850 \mu \mathrm{m}$ are $\sim 60$ and $\sim 10 \mathrm{mJy}$ beam $^{-1}$, respectively.

We use the level 2.5 Herschel/SPIRE $(250-500 \mu \mathrm{m})$ maps available in the Herschel Science Archive, ${ }^{38}$ using extended source calibration. The resolutions of the original maps at 250, 350 , and $500 \mu \mathrm{m}$ are approximately $18 . " 3,24 . " 9$, and $36 . " 3$, respectively.

\subsection{Line Observations}

Large-scale $\mathrm{C}^{18} \mathrm{O}(1-0)$ and ${ }^{13} \mathrm{CO}(1-0)$ are used to study the kinematics of the G035.39's natal molecular cloud. The $\mathrm{C}^{18} \mathrm{O}$ (1-0) and ${ }^{13} \mathrm{CO}(1-0)$ mapping data are obtained from the legacy survey program "TRAO Observations of PGCCs (TOP)" (Liu et al. 2018). The observations were conducted on 2017 March 17. The map size is $30^{\prime} \times 30^{\prime}$. The center of those maps is R.A.(J2000) $=18: 57: 10$, decl. $(\mathrm{J} 2000)=$ $+02: 10: 00$. The FWHM beam size $\left(\theta_{B}\right)$ is $47^{\prime \prime}$. The mainbeam efficiency $\left(\eta_{B}\right)$ is $51 \%$. The system temperature during observations is $243 \mathrm{~K}$. The OTF data were smoothed to $0.33 \mathrm{~km} \mathrm{~s}^{-1}$ and the baseline removed with Gildas/CLASS. The rms level is $0.15 \mathrm{~K}$ in antenna temperature $\left(T_{A}^{*}\right)$ at a spectral resolution of $0.33 \mathrm{~km} \mathrm{~s}^{-1}$.

Single-pointing observational data of the $\mathrm{HCO}^{+}(1-0)$, $\mathrm{H}^{13} \mathrm{CO}^{+}(1-0)$, and $\mathrm{H}_{2} \mathrm{CO}\left(2_{1,2}-1_{1,1}\right)$ lines are used to investigate the dynamical status of a starless clump in G035.39. The data taken with the Korean VLBI Network (KVN) $21 \mathrm{~m}$ telescope (Kim et al. 2011) in Tamna station were obtained on 2017 November 26 in its single-dish mode. The rest frequencies of $\mathrm{HCO}^{+}(1-0), \mathrm{H}^{13} \mathrm{CO}^{+}(1-0)$, and $\mathrm{H}_{2} \mathrm{CO}$ $\left(2_{1,2}-1_{1,1}\right)$ lines are $89.18852,86.754288$, and $140.83952 \mathrm{GHz}$, respectively. The pointing position is R.A.(J2000) = 18:57:11.38, decl.(J2000) $=+02: 07: 27.9$. The main-beam sizes at 86 and $140 \mathrm{GHz}$ are $32^{\prime \prime}$ and $23^{\prime \prime}$, respectively. The main-beam efficiencies at 86 and $140 \mathrm{GHz}$ are $44 \%$ and $36 \%$, respectively. The data are reduced with Gildas/CLASS. The spectral resolution for both the $\mathrm{HCO}^{+}(1-0)$ and $\mathrm{H}^{13} \mathrm{CO}^{+}(1-0)$ lines is $\sim 0.11 \mathrm{~km} \mathrm{~s}^{-1}$. The spectral resolution for $\mathrm{H}_{2} \mathrm{CO}$ $\left(2_{1,2}-1_{1,1}\right)$ is $0.07 \mathrm{~km} \mathrm{~s}^{-1}$. The on-source times for the $\mathrm{HCO}^{+}$ $(1-0), \mathrm{H}^{13} \mathrm{CO}^{+}(1-0)$, and $\mathrm{H}_{2} \mathrm{CO}\left(2_{1,2}-1_{1,1}\right)$ observations are 10,15 , and 25 minutes, respectively. The system temperatures

\footnotetext{
38 http://archives.esac.esa.int/hsa
} 


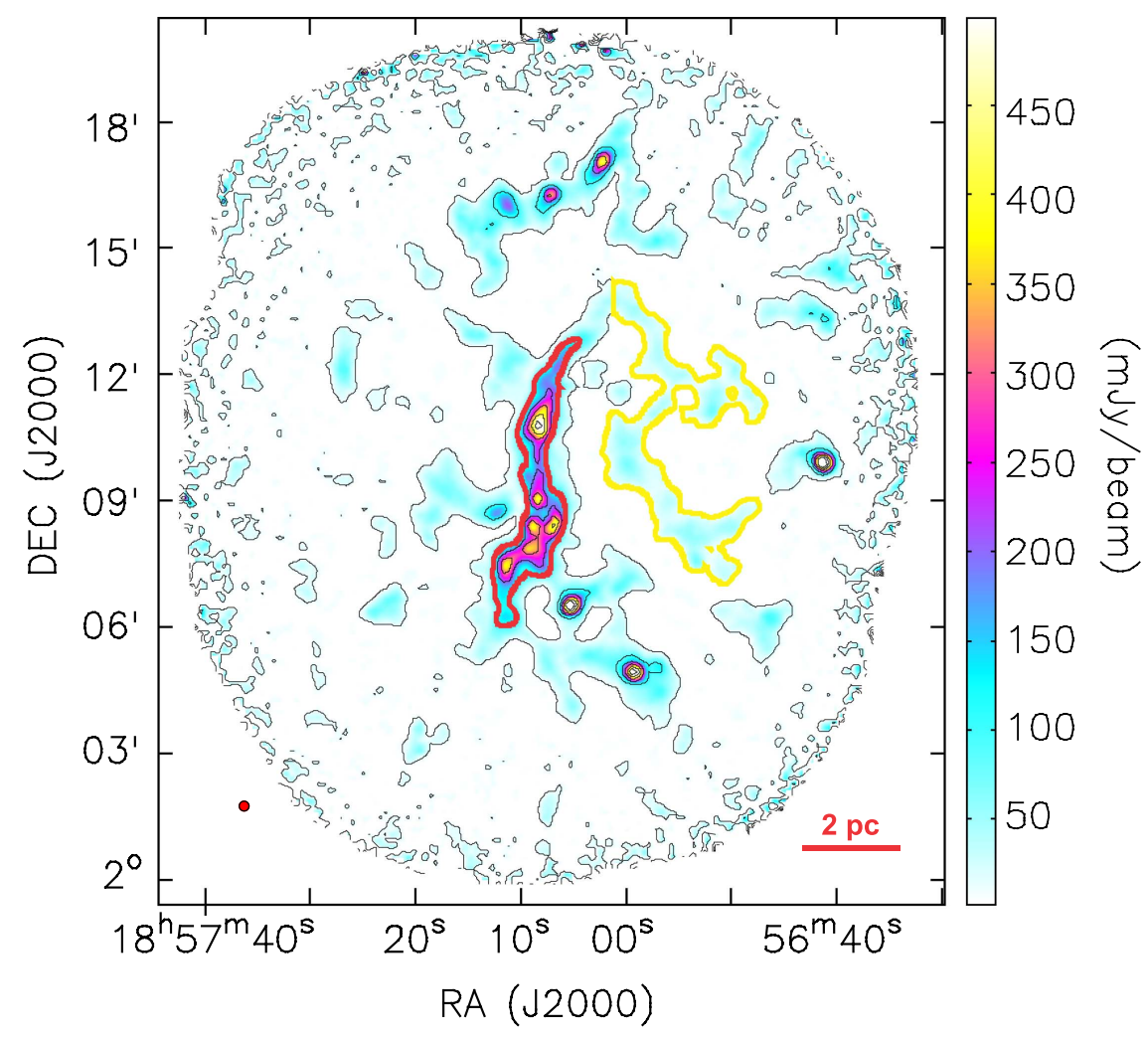

Figure 1. Stokes $I$ image at $850 \mu \mathrm{m}$ for G035.39. The outer contour level is $10 \mathrm{mJy}^{-1}$ beam $^{-1}$. The inner contours are from 100 to $500 \mathrm{mJy}^{-1}$ beam ${ }^{-1}$ in steps of $100 \mathrm{mJy}_{\text {beam }}{ }^{-1}$. The red contour $\left(100 \mathrm{mJy}\right.$ beam $\left.{ }^{-1}\right)$ outlines the main filament $F_{\mathrm{M}}$, and the yellow contour $\left(10 \mathrm{mJy}^{-1}\right.$ beam $\left.{ }^{-1}\right)$ outlines the faint western elongated structures $F_{\mathrm{W}}$. The red filled circle corresponds to the beam size.

during observations are 184,188 , and $171 \mathrm{~K}$, respectively. The achieved rms levels in antenna temperatures are $\sim 0.05, \sim 0.04$, and $\sim 0.03 \mathrm{~K}$, respectively.

We also use the $\mathrm{NH}_{3}(1,1)$ line data from Sokolov et al. (2017). The GBT beam at $\mathrm{NH}_{3}(1,1)$ line frequency is $32^{\prime \prime}$. The details of the $\mathrm{NH}_{3}(1,1)$ observations can be found in Sokolov et al. (2017).

\section{Results}

\subsection{Structure and Magnetic Field Geometry in G035.39}

Figure 1 shows the $850 \mu \mathrm{m}$ Stokes $I$ image. Besides the main filament (outlined with a red thick contour, hereafter denoted as $F_{\mathrm{M}}$ ) located at the center of the image, which was identified in previous work (Kainulainen \& Tan 2013), the deep SCUBA-2/POL-2 observations reveal several fainter adjacent elongated structures $\left(F_{\mathrm{W}}, F_{\mathrm{SW}}, F_{\mathrm{E}}\right.$, and $F_{\mathrm{NE}}$; see Figure 2) connected to $F_{\mathrm{M}}$. The skeletons of these elongated structures are identified by using the FILFINDER algorithm (Koch \& Rosolowsky 2015) in $850 \mu \mathrm{m}$ Stokes $I$ emission above $3 \sigma\left(1 \sigma \sim 1.5 \mathrm{mJy}\right.$ beam $\left.^{-1}\right)$. The skeletons are more easily identified in the high-contrast $850 \mu \mathrm{m}$ Stokes $I$ image than Herschel images because the extended diffuse emission is filtered out in SCUBA-2/POL-2 data. With larger filteringout scale, more extended emission can be recovered in $850 \mu \mathrm{m}$ Stokes $I$ data (Liu et al. 2018). Contamination from extended emission in $850 \mu \mathrm{m}$ continuum will reduce the contrast between the skeletons and the background emission. The FILFINDER algorithm adopting the techniques of mathematical morphology not only can identify the bright filaments but also can reliably extract a population of the faint filaments (Koch \& Rosolowsky 2015). The gray thick curves in Figure 2 show the skeletons of the elongated structures.

$F_{\mathrm{M}}$ has a length of $\sim 6.8 \mathrm{pc}$, measured from its skeleton. The longest elongated structure (outlined with a yellow thick contour in Figure 1; denoted as $\left.F_{\mathrm{W}}\right)$ having a similar length $(\sim 6.7 \mathrm{pc})$ to $F_{\mathrm{M}}$ is connected to the northern end of $F_{\mathrm{M}}$. The mean intensities of $F_{\mathrm{M}}$ and $F_{\mathrm{W}}$ at $850 \mu \mathrm{m}$ within the $10 \mathrm{mJy}$ beam ${ }^{-1}$ contours of the Stokes $I$ image are $\sim 100 \mathrm{mJy}_{\text {beam }}^{-1}$ and $\sim 24 \mathrm{mJy} \mathrm{beam}^{-1}$, respectively, suggesting that $F_{\mathrm{W}}$ is about four times fainter than $F_{\mathrm{M}}$.

The POS magnetic field orientations are shown in Figure 2. The field orientations are nearly perpendicular to the major axis of $F_{\mathrm{M}}$ at the middle ridge but tend to be parallel to its major axis at the lower-density tails. The field orientations of the elongated structures $\left(F_{\mathrm{SW}}, F_{\mathrm{E}}\right.$, and $\left.F_{\mathrm{NE}}\right)$ in their denser regions close to the junctions with $F_{\mathrm{M}}$ also tend to be perpendicular to their skeletons. In contrast, the field orientations associated with $F_{\mathrm{W}}$ are more parallel to its major axis. In this paper, we will mainly focus on $F_{\mathrm{M}}$. More detailed analysis and modeling of magnetic field geometry in the whole G035.39 field will be presented in a forthcoming paper (Juvela et al. 2018b).

Panel (a) in Figure 3 shows the magnetic field orientations associated with only $F_{\mathrm{M}}$. The magnetic field orientations are more disordered at the two ends and near the edges of the filament. In contrast, the magnetic field orientations become more ordered along the central spine of the filament. We average the orientations with a $16^{\prime \prime}$ pixel boxcar filter as Pattle et al. (2017) did and present the averaged orientations overlaid on a centroid velocity image of $\mathrm{NH}_{3}(1,1)$ from Sokolov et al. (2017) in panel (b) of Figure 3. We divide $F_{\mathrm{M}}$ into three regions ("N," "M," "S"), which show obvious differences in velocities and magnetic field geometries. "N" 


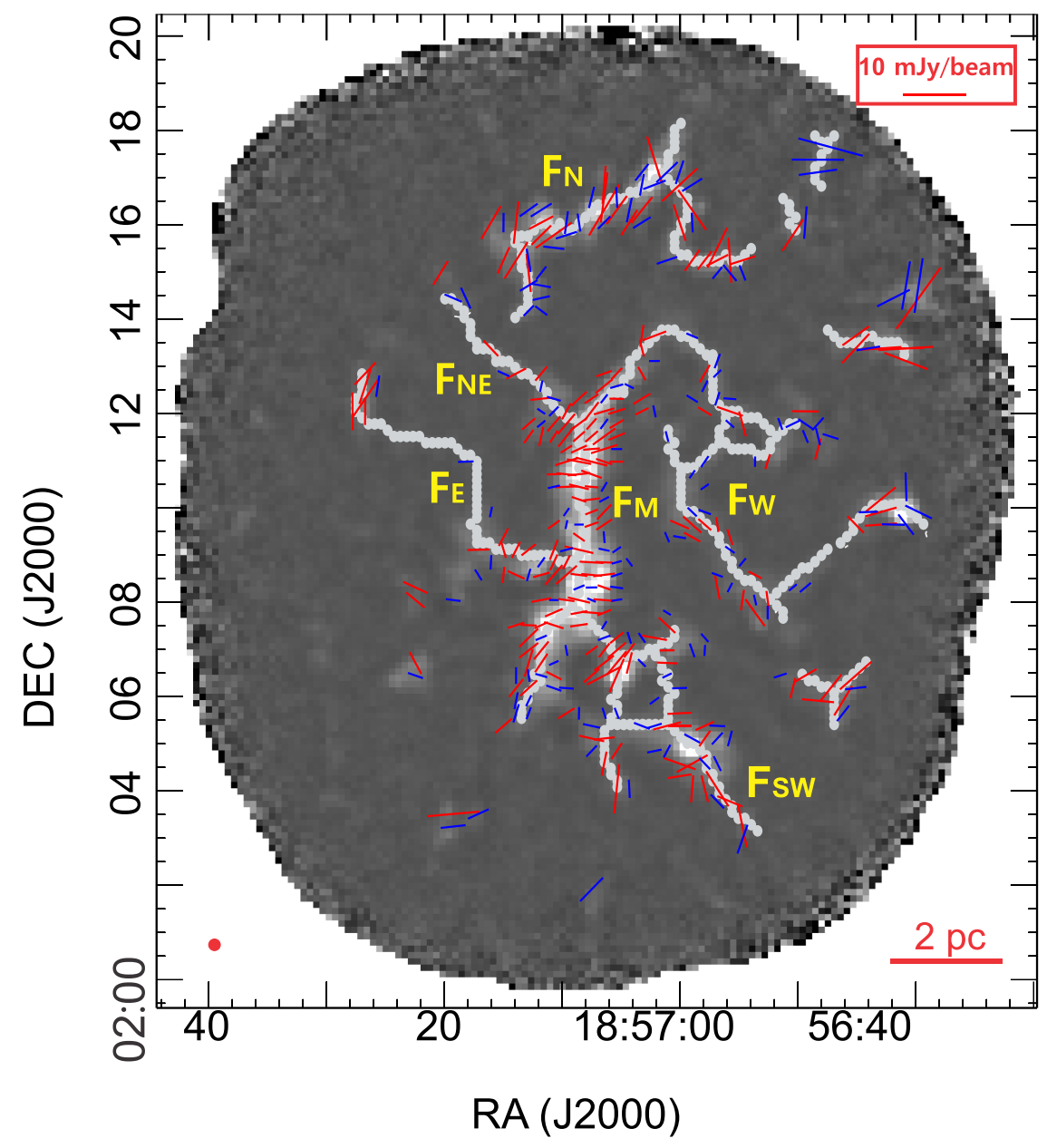

Figure 2. JCMT/POL-2 map of G035.39. The background is the Stokes $I$ image at $850 \mu \mathrm{m}$. The magnetic field orientations are averaged within $16^{\prime \prime}$ pixels. The red orientations are those detected at $\mathrm{S} / \mathrm{N}>3$ for polarization levels $(\mathrm{P})$. The blue orientations are $2<\mathrm{S} / \mathrm{N}<3$ for P. The cutoff for Stokes $I$ is $\mathrm{S} / \mathrm{N}>10$. The length of the orientations represents the polarization intensity in $\mathrm{mJy} \mathrm{beam}^{-1}$ (see scale bar). The gray thick curves show the skeletons of the elongated structures. The red filled circle corresponds to the beam size.

and "S" show redshifted and blueshifted line-of-sight velocities with respect to "M." In "N" and "S," the magnetic field orientations are more parallel to the filament skeletons. In contrast, the magnetic field orientations are more perpendicular to the filament skeletons in "M."

To investigate how the ordered magnetic field orientations change along the filament, we average the magnetic field orientations with a $32^{\prime \prime}$ pixel boxcar filter and calculate the angles $(\delta \theta)$ between the mean magnetic field orientations and the local orientations of the nearest skeletons. The boxcar filter has a size of $32^{\prime \prime}$ (or $0.45 \mathrm{pc}$ ), similar to the radius of the filament. The local orientations of the skeletons were measured from their gradients, similar to what Koch et al. (2012) did. The $\delta \theta$ as a function of offset distance from the northern end of $F_{\mathrm{M}}$ is presented in panel (c) of Figure 3. The magnetic field orientations in the end regions ("N" and " $\mathrm{S}$ ") are more parallel to the major axis of the filament with $\delta \theta \leqslant 40^{\circ}$. In contrast, the magnetic field orientations in the central part ("M") are more perpendicular to the major axis of the filament with $\delta \theta \geqslant 60^{\circ}$. From each end, $\delta \theta$ increases toward the middle part of $F_{\mathrm{M}}$.

Figure 4 presents histograms of the magnetic field orientations in the whole "M" region (green) and in the subregions (red and blue). The subregions are associated with dense clumps identified in Section 3.3. The blue histogram shows the position angles in the clump "c3" region. The red histogram shows the position angles in the region covering the clumps "c5," "c6," and "c7." Only the orientations with Stokes I intensity larger than $100 \mathrm{mJy}^{\text {beam }}{ }^{-1}$ are included. From a Gaussian fit, we obtain a mean magnetic field orientation of $\sim 86^{\circ}$ and an orientation dispersion $\left(\sigma_{\theta}\right)$ of $\sim 17^{\circ}$ in the whole "M" region. The mean magnetic field orientations $(\bar{\theta})$ in different subregions of the " $M$ " region are nearly the same, mostly perpendicular to the major axis of the filament.

Panel (d) of Figure 3 shows how the magnetic field orientation variation (with a default pixel size of $8^{\prime \prime}$ ) changes with Stokes $I$ intensity. The magnetic field orientations $(\theta)$ are subtracted by a mean value $\left(\bar{\theta}=86^{\circ}\right)$ in the densest part of "M." Although the uncertainties of orientations caused by noise have been considered in this plot, the orientation variations in low-density regions still show large dispersion, which can be improved by future higher-sensitivity observations. The orientation variation $|\theta-\bar{\theta}|$, however, is nearly constant toward higher Stokes $I$ intensity ( $>200 \mathrm{mJy}^{\text {beam }}{ }^{-1}$ ), suggesting that the magnetic field orientations in the central part of the $F_{\mathrm{M}}$ are more perpendicular to the major axis. In contrast, the orientation variations $|\theta-\bar{\theta}|$ become larger toward less dense regions (with Stokes $I$ intensity smaller than $100 \mathrm{mJy} \mathrm{beam}^{-1}$ ), suggesting that magnetic field orientations in less central regions tend to be more parallel to the major axis. 
(a)

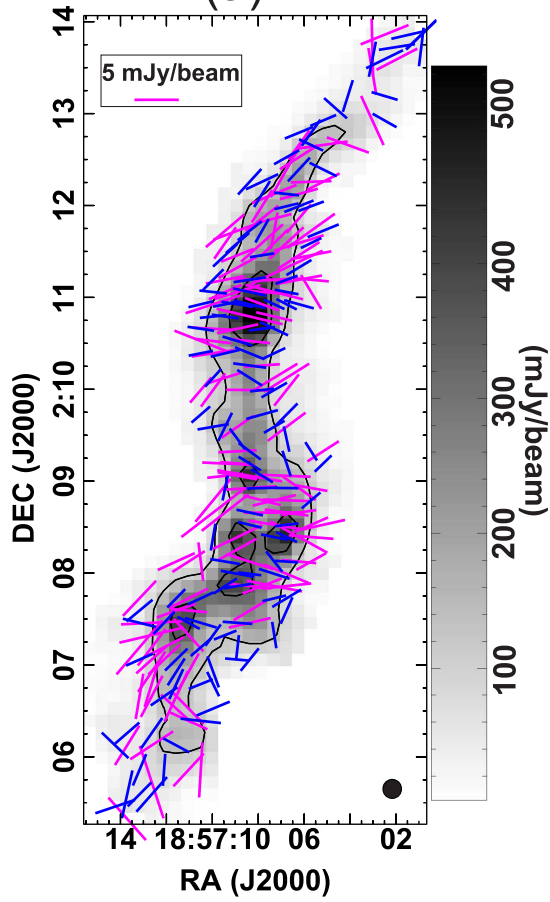

(b)

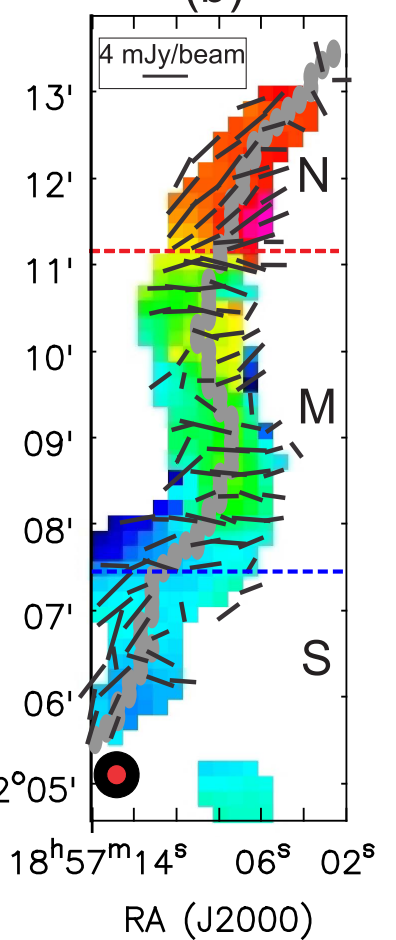

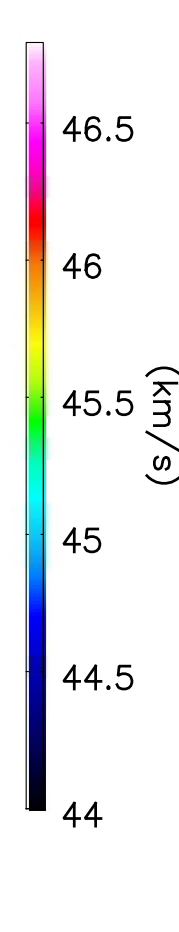

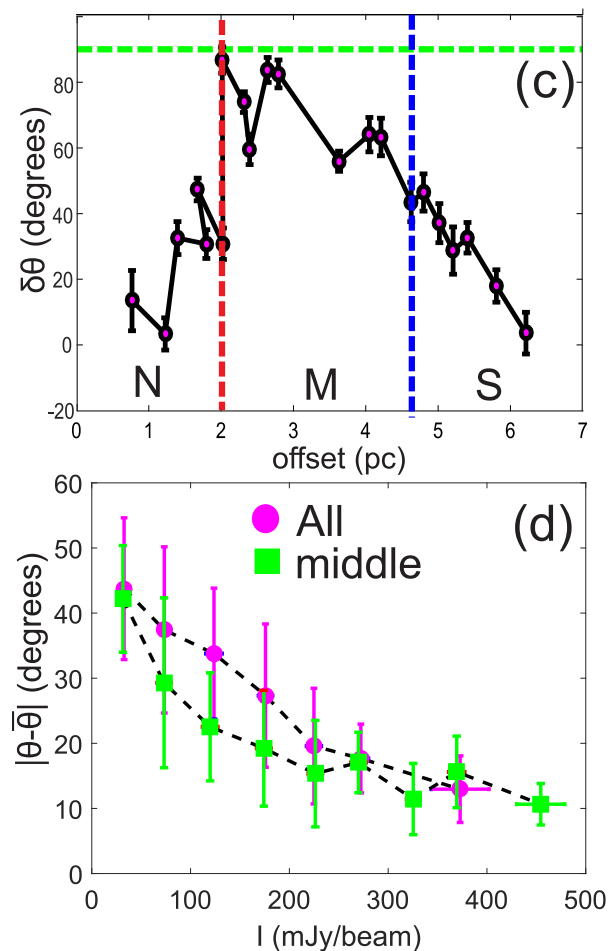

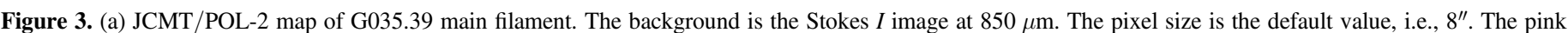

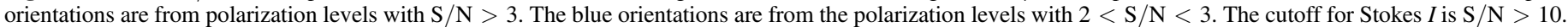

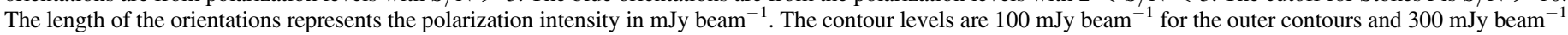

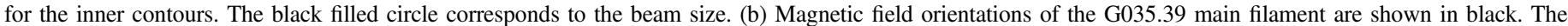

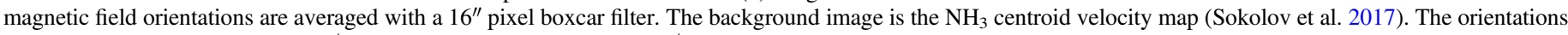

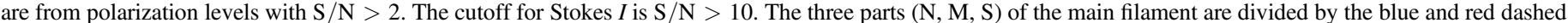

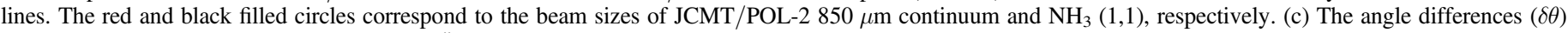

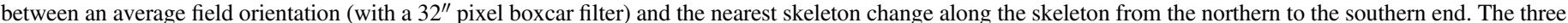

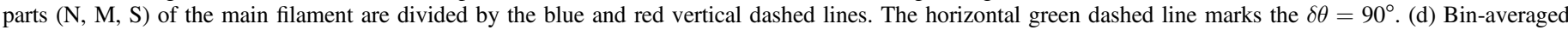

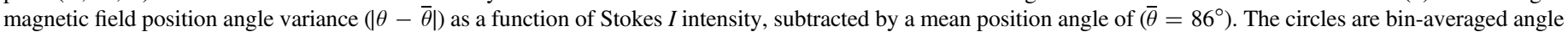
variations of orientations along the main filament. The boxes are bin-averaged angle variations of orientations in the middle part of the main filament.

\subsection{Properties of the Main Filament}

Column density $\left(N_{\mathrm{H}_{2}}\right)$ and dust temperature $\left(T_{\mathrm{d}}\right)$ maps of G035.39 are constructed from fitting the Herschel/SPIRE (250, 350 , and $500 \mu \mathrm{m})$ and JCMT/SCUBA-2 (450 and $850 \mu \mathrm{m})$ data with a modified blackbody (MBB) function, assuming a dust emissivity spectral index $(\beta)$ of 1.8 and the dust mass absorption coefficient $\kappa=0.1(\nu / 1 \mathrm{THz})^{\beta} \mathrm{cm}^{2} \mathrm{~g}^{-1} \quad$ (Juvela et al. 2018a). Only SCUBA-2 data at $450 \mu \mathrm{m}$ when the signal is above $150 \mathrm{MJy} \mathrm{sr}^{-1}$ and at $850 \mu \mathrm{m}$ when the signal is above $30 \mathrm{MJy} \mathrm{sr}{ }^{-1}$ in the $F_{\mathrm{M}}$ are used. In such compact, bright regions, SCUBA-2 data are not much affected by spatial filtering. Outside such regions, the SCUBA-2 data are corrected with an offset obtained after comparing an image convolved to the $40^{\prime \prime}$ resolution with a SPIRE-based prediction image at the same resolution.

The observations are fit with a model that consists of surface brightness maps at reference wavelengths and a color temperature map, all with a pixel size of $6^{\prime \prime}$. The model is fit to the observations as a global optimization problem. This involves the convolution of the MBB predictions of the model to the resolution of each of the observed surface brightness maps. After optimization, the final model is convolved to a resolution of $15^{\prime \prime}$, which is close to the resolution of the SCUBA-2 data. More details of the procedure are presented in a forthcoming paper (Juvela et al. 2018b). However, the results are found to be very close to those that would be obtained with the method described in the Appendix of Palmeirim et al. (2013), which similarly tries to maximize the resolution of the resulting column density maps. In our case, the dust temperature is constrained mainly by the SPIRE data, with the shortest wavelength $(250 \mu \mathrm{m})$ being close to the peak of the spectrum of cold dust emission. However, Juvela et al. (2012) estimated that even with $7 \%$ surface brightness errors, the SPIRE data give a better than $1 \mathrm{~K}$ accuracy for the temperatures (for $T \sim 15 \mathrm{~K}$ ), which corresponds only to $\sim 20 \%$ uncertainty in the column density. In the fits, we used the $4 \%$ and $10 \%$ error estimates for SPIRE and SCUBA-2 data, respectively.

The $N_{\mathrm{H}_{2}}$ and $T_{\mathrm{d}}$ maps of G035.39 are presented in Figure 5. Although the background emission is subtracted, the above method is still subject to the usual caveats with regard to the line-of-sight temperature variations (Juvela et al. 2018b). The mean dust temperature of $15 \mathrm{~K}$ that we derived, however, is very consistent with the mean dust temperature of $14 \mathrm{~K}$ (from a small median filter method) and mean kinetic temperature of $13 \mathrm{~K}$ derived by Sokolov et al. (2017).

The main filament $F_{\mathrm{M}}$ has much higher column density and is colder than its surroundings. The $N_{\mathrm{H} 2}$ and $T_{\mathrm{d}}$ maps of $F_{\mathrm{M}}$ are also presented in the right panel of Figure 5. Here we focus on the highest column density part of the main filament where 


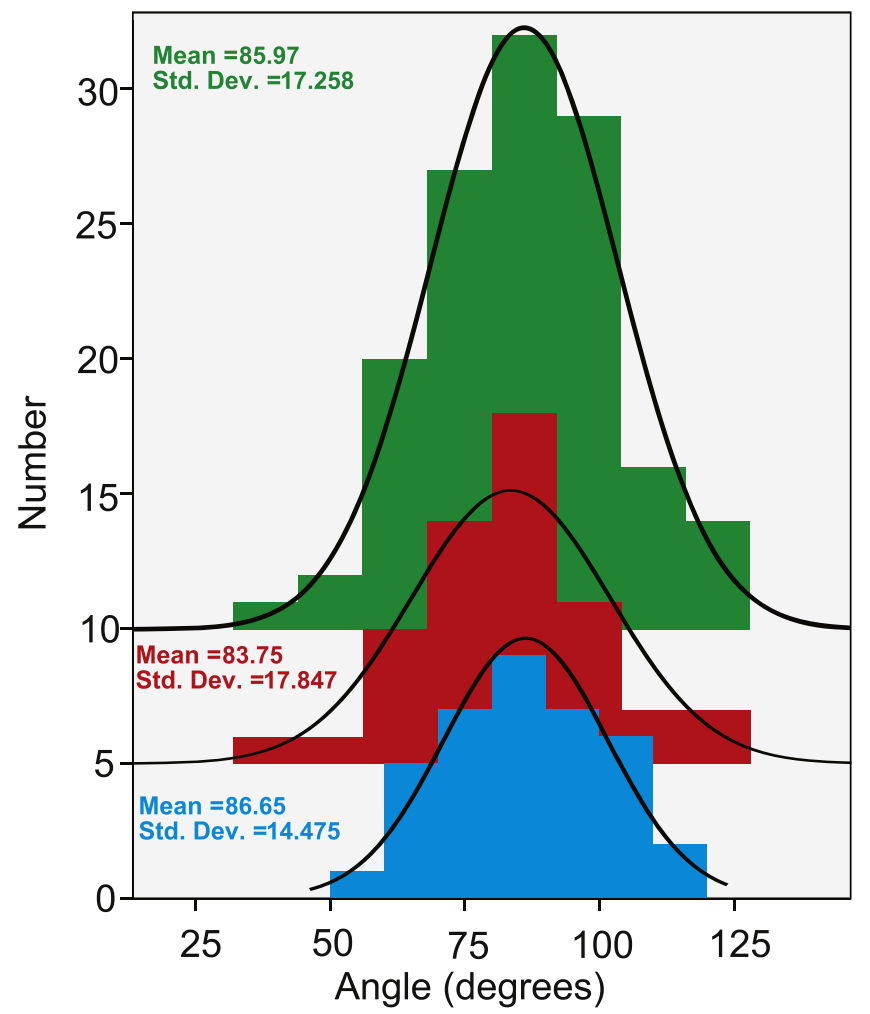

Figure 4. Histograms of magnetic field orientations in the middle ("M") part of the main filament. Only orientations with Stokes $I$ larger than $100 \mathrm{mJy}^{\text {beam }}{ }^{-1}$ are included in statistics. The histogram of magnetic field orientations in the whole middle part region is shown in green. The blue and red histograms are the magnetic field orientations in the subregions associated with dense clumps identified in Section 3.3. The blue histogram is the position angles in the clump "c3" region. The red histogram is the position angles in the region covering clumps "c5," "c6," and "c7." The black lines are Gaussian fits. The means and standard deviations (std. dev.) in the plots are obtained from Gaussian fitting.

$N_{\mathrm{H}_{2}}>7 \times 10^{21} \mathrm{~cm}^{-2}$, a column density threshold for core formation suggested in nearby clouds (André et al. 2014). The mean column density of the subregion is $\sim 1.8 \times$ $10^{22} \mathrm{~cm}^{-2}$. The length $(L)$ and the projected area $(A)$ of the ridge are $\sim 6.8$ and $\sim 6.9 \mathrm{pc}^{2}$, respectively. Therefore, the total mass $(M)$ of the subregion is calculated as

$$
M=N_{\mathrm{H}_{2}} \times A \mu_{g} m_{\mathrm{H}}
$$

where $\mu_{g}=2.8$ is the molecular weight per hydrogen molecule and $m_{\mathrm{H}}$ is the mass of a hydrogen atom. The derived mass is $\sim 2800 M_{\odot}$. Therefore, the line mass (mass per unit length) of the filament is $M / L \sim 410 M_{\odot} \mathrm{pc}^{-1}$, which is within the range (223-635 $M_{\odot} \mathrm{pc}^{-1}$ ) given by Sokolov et al. (2017). Assuming that the filament has cylindrical geometry, the mean radius $(r)$ of the circular end of the cylinder is

$$
r=\frac{A}{2 L} \approx 0.5 \mathrm{pc}
$$

where $A$ is the projected area of the filament. Therefore, the volume $(V)$ of the cylindrical filament is

$$
V=\pi r^{2} \times L \approx 5.4 \mathrm{pc}^{3},
$$

and the mean volume density $\left(N_{\mathrm{H} 2}\right)$ is

$$
n_{\mathrm{H}_{2}}=\frac{M}{V \mu_{g} m_{\mathrm{H}}} \approx 7.3 \times 10^{3} \mathrm{~cm}^{-3} .
$$

In contrast, the average column density and dust temperature of the fainter, western elongated structures $F_{\mathrm{W}}$ are $4.0 \times 10^{21} \mathrm{~cm}^{-2}$ and $\sim 21 \mathrm{~K}$, respectively, which are calculated within the $10 \mathrm{mJy}^{-1}$ beam ${ }^{-1}$ contours of the Stokes $I$ intensity at $850 \mu \mathrm{m}$. It is noted, however, that the column density and dust temperature of $F_{\mathrm{W}}$ are less constrained than those of $F_{\mathrm{M}}$ because only Herschel data are used in SEDs fit toward $F_{\mathrm{W}}$. The mass of $F_{\mathrm{W}}$ is $\sim 650 M_{\odot}$. Considering the length $(\sim 6.7 \mathrm{pc})$ of $F_{\mathrm{W}}$, its line mass and volume density are $\sim 100 M_{\odot} \mathrm{pc}^{-1}$ and $1.7 \times 10^{3} \mathrm{~cm}^{-3}$, respectively. The total mass, line mass, column density, and volume density of $F_{\mathrm{W}}$ are all about four times smaller than those of $F_{\mathrm{M}}$.

\subsection{Fragmentation of the Main Filament}

The main filament $F_{\mathrm{M}}$ shows a chain of clumps with nearly even spacing. As shown in the right panel of Figure 5, we extracted nine dense clumps from the SCUBA-2 $850 \mu \mathrm{m}$ image using the FELLWALKER (Berry 2015) source-extraction algorithm, which is a part of the Starlink CUPID package (Berry 2007). The core of the FELLWALKER algorithm is a gradient-tracing scheme that follows many different paths of steepest ascent in order to reach a significant summit, each of which is associated with a clump (Berry 2007). FELLWALKER is less dependent on specific parameter settings than other source-extraction algorithms (e.g., CLUMPFIND; Berry 2007).

The source-extraction process with FELLWALKER is the same as that used by the JCMT Plane Survey, and the details can be found in Moore et al. (2015) and Eden et al. (2017). A mask constructed above a threshold of $3 \sigma$ in the signal-to-noise ratio $(\mathrm{S} / \mathrm{N})$ map is applied to the intensity map as input for the task CUPID:EXTRACTCLUMPS, which extracts the peak and integrated flux density values of the clumps. A further threshold for CUPID:FINDCLUMPS is the minimum number of contiguous pixels, which is set at 12 , corresponding to the number of pixels expected to be found in an unresolved source with a peak $\mathrm{S} / \mathrm{N}$ of $5 \sigma$, given a $14^{\prime \prime}$ beam and $4^{\prime \prime}$ pixels.

The coordinates, radii $\left(R_{\text {eff }}\right)$, mean dust temperature $\left(T_{d}\right)$, mean column density $\left(N_{\mathrm{H}_{2}}\right)$, and mean nonthermal velocity dispersion $\left(\sigma_{\mathrm{NT}}\right)$ of the clumps derived from $\mathrm{NH}_{3}(1,1)$ line emission are presented in Table 1. The mean separation between clumps is $\sim 0.9 \mathrm{pc}$. The effective radii of clumps are $R_{\text {eff }}=\sqrt{a b}$, where $a$ and $b$ are the sizes of the semimajor axis and semiminor axis of the clump from a 2D Gaussian fit, respectively. The beam-deconvolved effective radii $\left(R_{\text {eff }}\right)$ of clumps range from 0.12 to $0.31 \mathrm{pc}$, with an average value of $0.23 \mathrm{pc}$. The dust temperatures of the clumps range from 13.2 to $17.0 \mathrm{~K}$, with an average value of $14.6 \mathrm{~K}$. Clump masses $\left(M_{\text {clump }}\right)$ are derived as

$$
M_{\text {clump }}=\pi R_{\text {eff }}^{2} N_{\mathrm{H}_{2}} \mu_{g} m_{\mathrm{H}} .
$$

Assuming that the clumps have spherical geometry, their $\mathrm{H}_{2}$ volume density $\left(n_{\mathrm{H}_{2}}\right)$ is derived as

$$
n_{\mathrm{H}_{2}}=\frac{M}{\frac{4}{3} \pi R_{\mathrm{eff}}^{3} \mu_{g} m_{\mathrm{H}}} .
$$


(a)

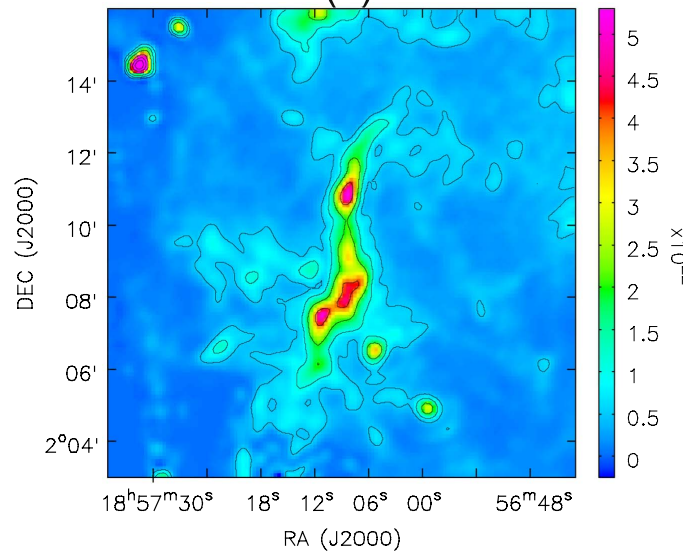

(b)

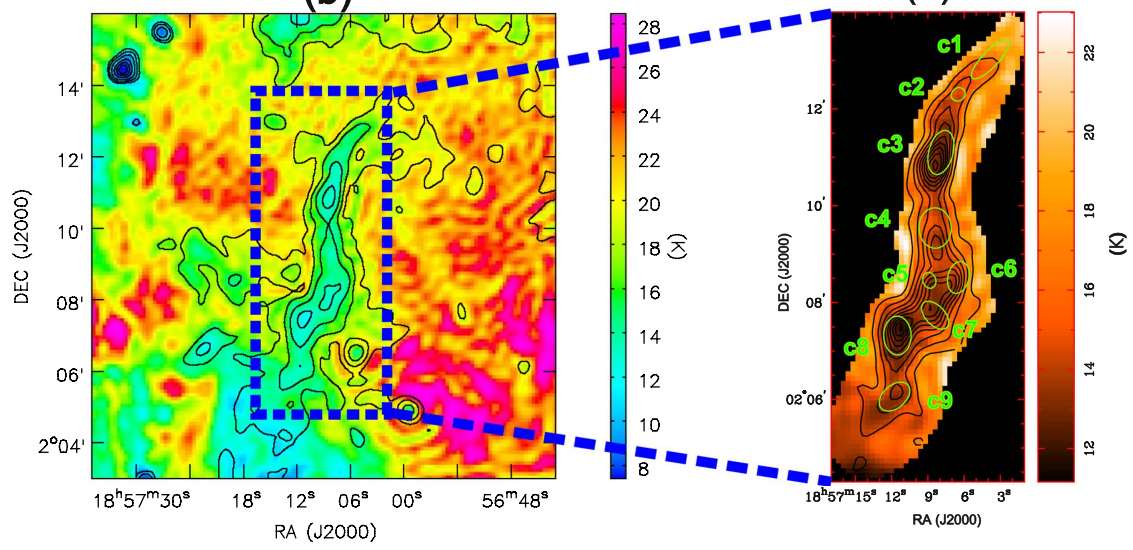

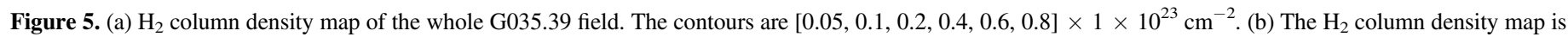

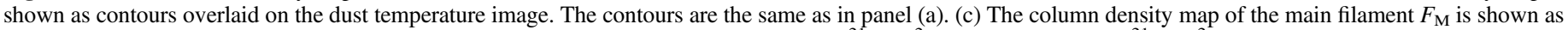

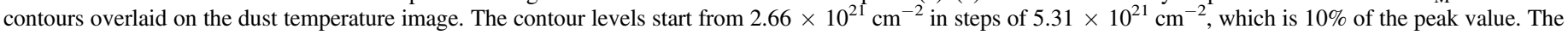
clumps identified via FELLWALKER are shown with green ellipses.

The volume densities and masses of clumps are also presented in Table 1. The masses of clumps range from 16 to $219 M_{\odot}$, with a mean value of $\sim 107 M_{\odot}$. The volume densities of clumps range from $8 \times 10^{3} \mathrm{~cm}^{-3}$ to $6.4 \times 10^{4} \mathrm{~cm}^{-3}$, with a mean value of $3 \times 10^{4} \mathrm{~cm}^{-3}$.

\subsection{Large-scale Distribution of the Gas}

Panel (a) of Figure 6 shows the averaged spectrum of the ${ }^{13} \mathrm{CO}(1-0)$ line emission toward the main filament $F_{\mathrm{M}}$ (enclosed by the green contour in panels (c) to (g) of Figure 6). Multiple velocity components are seen in ${ }^{13} \mathrm{CO}$ (1-0) line emission, suggesting that several clouds are overlapped along the line of sight. Those velocity components are well separated by at least $10 \mathrm{~km} \mathrm{~s}^{-1}$. The four strongest components can be well fitted with Gaussian functions. Their peak velocities, line widths, and brightness temperature are listed in Table 2. The emission around $90 \mathrm{~km} \mathrm{~s}^{-1}$ is weak and was not fitted.

Panel (b) of Figure 6 shows the averaged spectrum of the ${ }^{13} \mathrm{CO}(1-0)$ line emission toward the western elongated structures $F_{\mathrm{W}}$ (enclosed by the magenta contour in panels (c) to (g) of Figure 6). Five velocity components are seen in the ${ }^{13} \mathrm{CO}$ (1-0) line emission. We fit four components with Gaussian functions and summarize the fitting parameters in Table 2. The emission (highlighted in red) between 50 and $70 \mathrm{~km} \mathrm{~s}^{-1}$ contains a narrow component and a broad component. The broad component overlaps in velocity with the $45.4 \mathrm{~km} \mathrm{~s}^{-1}$ component.

Panels (c) $-(\mathrm{g})$ of Figure 6 present the integrated intensity maps of each velocity component in ${ }^{13} \mathrm{CO}(1-0)$ line emission. The structures identified in $850 \mu \mathrm{m}$ continuum emission are mainly associated with the velocity feature at $45 \mathrm{~km} \mathrm{~s}^{-1}$ (see panel (e)). Previous molecular line studies have also suggested that the main filament $F_{\mathrm{M}}$ is dominated by emission around $45 \mathrm{~km} \mathrm{~s}^{-1}$ (Sanhueza et al. 2012; Henshaw et al. 2013, 2014, 2017; Jiménez-Serra et al. 2014; Sokolov et al. 2017). The emission around $13 \mathrm{~km} \mathrm{~s}^{-1}$ (see panel (c)) is very diffuse and is mainly distributed to the west of $F_{\mathrm{M}}$. The emission around $27 \mathrm{~km} \mathrm{~s}^{-1}$ (see panel (d)) is mainly located to the north of $F_{\mathrm{M}}$ and is partially overlapped with $F_{\mathrm{M}}$. The emission around $93 \mathrm{~km} \mathrm{~s}^{-1}$ (see panel $(\mathrm{g})$ ) is overlapped with $F_{\mathrm{M}}$ and $F_{\mathrm{W}}$ but shows a very large velocity difference with respect to the $45 \mathrm{~km} \mathrm{~s}^{-1}$ component, suggesting that the $93 \mathrm{~km} \mathrm{~s}^{-1}$ component is not really physically associated with $F_{\mathrm{M}}$ and $F_{\mathrm{W}}$. Similar to the $45 \mathrm{~km} \mathrm{~s}^{-1}$ component, the emission around $56 \mathrm{~km} \mathrm{~s}^{-1}$ (see panel (f)) also shows filamentary structures. Its emission, however, is mainly distributed in the north and northwest of $F_{\mathrm{M}}$ and $F_{\mathrm{W}}$. A long filament (marked by the yellow dashed line in panel (f)) in the $56 \mathrm{~km} \mathrm{~s}^{-1}$ cloud overlaps with the southern part of $F_{\mathrm{M}}$. Since the velocity difference between the $56 \mathrm{~km} \mathrm{~s}^{-1}$ component and the $45 \mathrm{~km} \mathrm{~s}^{-1}$ component is larger than $10 \mathrm{~km} \mathrm{~s}^{-1}, F_{\mathrm{M}}$ and $F_{\mathrm{W}}$ may not be greatly affected by the $56 \mathrm{~km} \mathrm{~s}^{-1}$ cloud. The $56 \mathrm{~km} \mathrm{~s}^{-1}$ cloud, however, is adjacent to the west part (marked by the green dashed line in panel (e)) of the $45 \mathrm{~km} \mathrm{~s}^{-1}$ cloud and shows broad emission therein (see panel (b)), which suggests that the $56 \mathrm{~km} \mathrm{~s}^{-1}$ cloud may be interacting with the $45 \mathrm{~km} \mathrm{~s}^{-1}$ cloud. The $45 \mathrm{~km} \mathrm{~s}^{-1}$ cloud shows two elongated structures as shown by the dashed lines in panel (e). The main part, which contains $F_{\mathrm{M}}$, has a length of at least $\sim 20 \mathrm{pc}$. The western part, which contains $F_{\mathrm{W}}$, has a length of $\sim 17 \mathrm{pc}$.

Panel (a) of Figure 7 presents the averaged spectra of $\mathrm{C}^{18} \mathrm{O}$ (1-0) line emission in the $F_{\mathrm{M}}$ and $F_{\mathrm{W}}$ regions. The $\mathrm{C}^{18} \mathrm{O}(1-0)$ line emission also shows two velocity components at $\sim 45 \mathrm{~km} \mathrm{~s}^{-1}$ (see panel (b)) and $\sim 56 \mathrm{~km} \mathrm{~s}^{-1}$ (see panel (c)). The $\sim 56 \mathrm{~km} \mathrm{~s}^{-1}$ component, however, is very weak and marginally detected toward $F_{\mathrm{M}}$ and $F_{\mathrm{W}}$. Panels (b) and (c) present the integrated intensity maps of the two velocity components. $F_{\mathrm{M}}$ is clearly associated with the $45 \mathrm{~km} \mathrm{~s}^{-1}$ emission but offset from the $\sim 56 \mathrm{~km} \mathrm{~s}^{-1}$ emission. As shown in panel (d), the $\mathrm{C}^{18} \mathrm{O}(1-0)$ line emission around $45 \mathrm{~km} \mathrm{~s}^{-1}$ has similar morphology to the $850 \mu$ m continuum emission. $F_{\mathrm{W}}$ is not obviously seen in $\mathrm{C}^{18} \mathrm{O}(1-0)$ emission maps. The $\mathrm{C}^{18} \mathrm{O}$ (1-0) emission signal is marginally detected as seen from the averaged spectrum in panel (a). From Gaussian fitting, the $\mathrm{C}^{18} \mathrm{O}(1-0)$ line luminosity of $F_{\mathrm{W}}$ is about five times smaller than that of $F_{\mathrm{M}} . F_{\mathrm{W}}$ and $F_{\mathrm{M}}$, however, show very similar velocity $\left(\sim 44.9 \pm 0.1 \mathrm{~km} \mathrm{~s}^{-1}\right.$ for both) and line widths $(1.8 \pm$ $0.4 \mathrm{~km} \mathrm{~s}^{-1}$ for $F_{\mathrm{W}}$ and $2.1 \pm 0.1 \mathrm{~km} \mathrm{~s}^{-1}$ for $\left.F_{\mathrm{M}}\right)$ in $\mathrm{C}^{18} \mathrm{O}(1-0)$ emission, suggesting that $F_{\mathrm{W}}$ and $F_{\mathrm{M}}$ are kinematically and spatially connected.

$F_{\mathrm{W}}$ has ${ }^{13} \mathrm{CO}(1-0)$ and $\mathrm{C}^{18} \mathrm{O}(1-0)$ line widths similar to those of $F_{\mathrm{M}} \cdot F_{\mathrm{W}}$, however, is about four times less dense than 
Table 1

Parameters of Dense Clumps in the Main Filament

\begin{tabular}{|c|c|c|c|c|c|c|c|c|c|c|c|c|c|}
\hline $\begin{array}{l}\text { Clump } \\
\text { No. }\end{array}$ & $\begin{array}{l}\text { R.A. } \\
\text { (J2000) }\end{array}$ & $\begin{array}{l}\text { Decl. } \\
(\mathrm{J} 2000)\end{array}$ & $\begin{array}{l}R_{\text {eff }}^{\mathrm{a}} \\
(\mathrm{pc})\end{array}$ & $\begin{array}{l}T_{\mathrm{d}}^{\mathrm{b}} \\
(\mathrm{K})\end{array}$ & $\begin{array}{c}N_{\mathrm{H}_{2}}{ }^{\mathrm{c}}{ }^{-2} \\
\left(10^{22} \mathrm{~cm}^{-2}\right)\end{array}$ & $\begin{array}{c}\sigma_{\mathrm{NT}}^{\mathrm{d}} \\
\left(\mathrm{km} \mathrm{s}^{-1}\right)\end{array}$ & $\begin{array}{c}n_{\mathrm{H}_{2}} \\
\left(10^{4} \mathrm{~cm}^{-3}\right)\end{array}$ & $\begin{array}{c}B_{\text {clump }} \\
(\mu \mathrm{G})\end{array}$ & $\begin{array}{c}\sigma_{A} \\
\left(\mathrm{~km} \mathrm{~s}^{-1}\right)\end{array}$ & $\mathcal{M}_{\mathcal{A}}$ & $\begin{array}{c}M_{\text {clump }} \\
\left(M_{\odot}\right)\end{array}$ & $\begin{array}{l}M_{\text {vir }} \\
\left(M_{\odot}\right)\end{array}$ & $\begin{array}{l}M_{\text {vir }}^{B} \\
\left(M_{\odot}\right)\end{array}$ \\
\hline c1 & $18: 57: 03.84$ & $+02: 13: 01.2$ & 0.25 & 17.0 & 0.8 & 0.31 & 0.8 & 56 & 0.8 & 0.6 & 35 & 45 & 78 \\
\hline c2 & $18: 57: 06.48$ & $+02: 12: 18.0$ & 0.12 & 14.6 & 1.6 & 0.30 & 3.2 & 142 & 1.0 & 0.5 & 16 & 19 & 44 \\
\hline c3 & 18:57:07.92 & $+02: 11: 06.0$ & 0.29 & 14.7 & 3.7 & 0.47 & 3.1 & 138 & 1.0 & 0.8 & 219 & 92 & 150 \\
\hline c4 & $18: 57: 08.40$ & $+02: 09: 32.4$ & 0.31 & 15.0 & 2.2 & 0.45 & 1.7 & 94 & 0.9 & 0.8 & 149 & 92 & 144 \\
\hline$c 5$ & 18:57:08.88 & $+02: 08: 27.6$ & 0.13 & 14.0 & 3.4 & 0.70 & 6.4 & 219 & 1.1 & 1.1 & 40 & 81 & 114 \\
\hline c6 & $18: 57: 06.48$ & $+02: 08: 31.2$ & 0.23 & 15.4 & 2.8 & 0.47 & 3.0 & 133 & 1.0 & 0.8 & 104 & 73 & 119 \\
\hline c9 & $18: 57: 11.76$ & $+02: 06: 03.6$ & 0.25 & 13.9 & 1.7 & 0.34 & 1.7 & 91 & 0.9 & 0.6 & 75 & 45 & 89 \\
\hline
\end{tabular}

Notes.

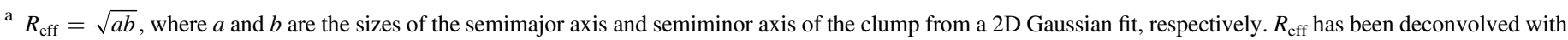
the beam.

${ }^{\mathrm{b}}$ Clump-averaged dust temperature $\left(T_{\mathrm{d}}\right)$.

c Mean column density.

${ }^{\mathrm{d}}$ Clump-averaged velocity dispersion derived from line widths of $\mathrm{NH}_{3}(1,1)$ emission (Sokolov et al. 2017).

$F_{\mathrm{M}}$, suggesting that turbulence in $F_{\mathrm{W}}$ likely plays a relatively more important role compared to $F_{\mathrm{M}}$.

Table 2 summarizes the parameters of the averaged spectra of ${ }^{13} \mathrm{CO}(1-0)$ and $\mathrm{C}^{18} \mathrm{O}(1-0)$ line emission toward $F_{\mathrm{M}}$ and $F_{\mathrm{W}}$, including the centroid velocities, line widths, and peak brightness temperature from the Gaussian fitting. Their mean dust temperatures, column densities, and $\mathrm{H}_{2}$ number densities are also listed. The column densities of ${ }^{13} \mathrm{CO}(1-0)$ and $\mathrm{C}^{18} \mathrm{O}(1-0)$ lines are derived using the non-LTE radiation transfer code, RADEX (Van der Tak et al. 2007). The inputs for RADEX are kinetic temperature $\left(T_{\mathrm{k}}\right), \mathrm{H}_{2}$ number density $\left(n_{\mathrm{H}_{2}}\right)$, molecular line column density $\left(N_{\text {line }}\right)$, and line width $(\Delta v)$. We assume that the $T_{\mathrm{k}}$ is equal to the dust temperature. By fixing $T_{\mathrm{k}}, n_{\mathrm{H}_{2}}$, and $\Delta v$, we can calculate the brightness temperature for a grid of $N_{\text {line }}$ values and compare the model brightness temperature with observed values to find out the best $N_{\text {line }}$. The derived molecular line column densities and abundances are listed also in Table 2. The mean column densities of ${ }^{13} \mathrm{CO}$ in $F_{\mathrm{M}}$ and $F_{\mathrm{W}}$ are $\sim 2.2 \times 10^{16} \mathrm{~cm}^{-2}$ and $\sim 8.5 \times 10^{15} \mathrm{~cm}^{-2}$, respectively. The mean column densities of $\mathrm{C}^{18} \mathrm{O}$ in $F_{\mathrm{M}}$ and $F_{\mathrm{W}}$ are $\sim 2.6 \times 10^{15} \mathrm{~cm}^{-2}$ and $\sim 4.5 \times 10^{14} \mathrm{~cm}^{-2}$, respectively. The ${ }^{13} \mathrm{CO}$ abundance in $F_{\mathrm{M}}$ is about two times smaller than that in $F_{\mathrm{W}}$, possibly due to larger opacity in ${ }^{13} \mathrm{CO}(1-0)$ emission in $F_{\mathrm{M}}$. In contrast, $F_{\mathrm{M}}$ and $F_{\mathrm{W}}$ have very similar $\mathrm{C}^{18} \mathrm{O}$ abundances. By comparing the observed $\mathrm{C}^{18} \mathrm{O}$ abundance $\left(X_{\text {obs }}\right)$ with a canonical $\mathrm{C}^{18} \mathrm{O}$ abundance $\left(X_{\text {cano }} \sim 6.1 \times 10^{-7}\right)$, Jiménez-Serra et al. (2014) claimed high CO depletion factors $\left(f_{\mathrm{D}}=X_{\text {cano }} / X_{\mathrm{obs}} \sim 5-12\right)$ in all three regions of $F_{\mathrm{M}}$. We derive a mean $\mathrm{CO}$ depletion factor of $\sim 6$ for both $F_{\mathrm{M}}$ and $F_{\mathrm{W}}$ if we adopt the same canonical $\mathrm{C}^{18} \mathrm{O}$ abundance. $F_{\mathrm{M}}$ is much denser than $F_{\mathrm{W}}$, and thus $\mathrm{CO}$ may be expected to be more depleted in $F_{\mathrm{M}}$. The $\mathrm{C}^{18} \mathrm{O}$ and ${ }^{13} \mathrm{CO}$ abundances in both $F_{\mathrm{M}}$ and $F_{\mathrm{W}}$, however, only vary by a factor of $1-2$ in our observations, suggesting that $\mathrm{CO}$ in $F_{\mathrm{M}}$ may not be depleted as severely as suggested in previous studies (e.g., Jiménez-Serra et al. 2014). Observations in Jiménez-Serra et al. (2014) have much better spatial resolution than ours, and thus they arguably trace better the inner parts of the filament. Therefore, high CO depletion may occur in densest regions of the filament. The uncertainties of the adopted canonical abundance, however, may prevent an accurate determination of $\mathrm{CO}$ depletion levels because observed CO abundances vary cloud by cloud in the Galaxy (Liu et al. 2013c; Giannetti et al. 2014). Finally, the optical depths and excitation temperatures are listed in Table 2 . The averaged $\mathrm{C}^{18} \mathrm{O}$ $(1-0)$ and ${ }^{13} \mathrm{CO}(1-0)$ lines are optically thin for both $F_{\mathrm{M}}$ and $F_{\mathrm{W}}$. The excitation temperatures of $\mathrm{C}^{18} \mathrm{O}(1-0)$ and ${ }^{13} \mathrm{CO}(1-0)$ in $F_{\mathrm{M}}$. are $\sim 15.0$ and $14.1 \mathrm{~K}$, respectively. Being similar to the dust temperature, these temperatures suggest that $\mathrm{C}^{18} \mathrm{O}$ and ${ }^{13} \mathrm{CO}$ lines in $F_{\mathrm{M}}$ are nearly thermally excited and that local thermodynamical equilibrium conditions hold. The excitation temperatures of the $\mathrm{C}^{18} \mathrm{O}(1-0)$ and ${ }^{13} \mathrm{CO}(1-0)$ line emissions in $F_{\mathrm{W}}$, however, are $\sim 14.3$ and $14.5 \mathrm{~K}$, respectively, which are lower than the dust temperature $(\sim 21 \mathrm{~K})$, suggesting that $\mathrm{C}^{18} \mathrm{O}(1-0)$ and ${ }^{13} \mathrm{CO}(1-0)$ lines in $F_{\mathrm{W}}$ are subthermally excited.

\section{Discussion \\ 4.1. Massive Filament Formed owing to Cloud-Cloud Collision}

A large-scale, smooth velocity gradient of $0.4-0.8 \mathrm{~km} \mathrm{~s}^{-1} \mathrm{pc}^{-1}$ in the northern part of the main filament $\left(F_{\mathrm{M}}\right)$ has been revealed in the ${ }^{13} \mathrm{CO}, \mathrm{C}^{18} \mathrm{O}$, and $\mathrm{N}_{2} \mathrm{H}^{+}$line emissions (Henshaw et al. 2014; Jiménez-Serra et al. 2014). Recently, Sokolov et al. (2017) mapped the whole $F_{\mathrm{M}}$ in $\mathrm{NH}_{3}$ lines and also found a smooth velocity gradient of $\sim 0.2 \mathrm{~km} \mathrm{~s}^{-1} \mathrm{pc}^{-1}$ across the whole filament as shown in panel (b) of Figure 3. Several scenarios have been proposed to explain these gradients, including cloud rotation, gas accretion along the filaments, global gravitational collapse, and unresolved subfilament structures (Jiménez-Serra et al. 2014). A very promising scenario that could explain the presence of the smooth velocity gradient would involve the initial formation of substructures inside the turbulent molecular cloud, which interact with each other and may subsequently converge into each other as the cloud undergoes global gravitational collapse (Jiménez-Serra et al. 2014). From high-sensitivity and high spectral resolution molecular line $\left(\mathrm{N}_{2} \mathrm{H}^{+}\right.$and $\left.\mathrm{C}^{18} \mathrm{O}\right)$ observations, Henshaw et al. (2013) identify several velocity-coherent filaments inside $F_{\mathrm{M}}$ and argue that $F_{\mathrm{M}}$ formed via the collision of two relatively quiescent filaments moving at a relative velocity of $\sim 5 \mathrm{~km} \mathrm{~s}^{-1}$.

Based on our large-scale ${ }^{13} \mathrm{CO}(1-0)$ map, we argue here that the velocity gradients and the collision of filaments inside $F_{\mathrm{M}}$ are more likely caused by a large-scale $(\sim 10 \mathrm{pc})$ cloud-cloud 

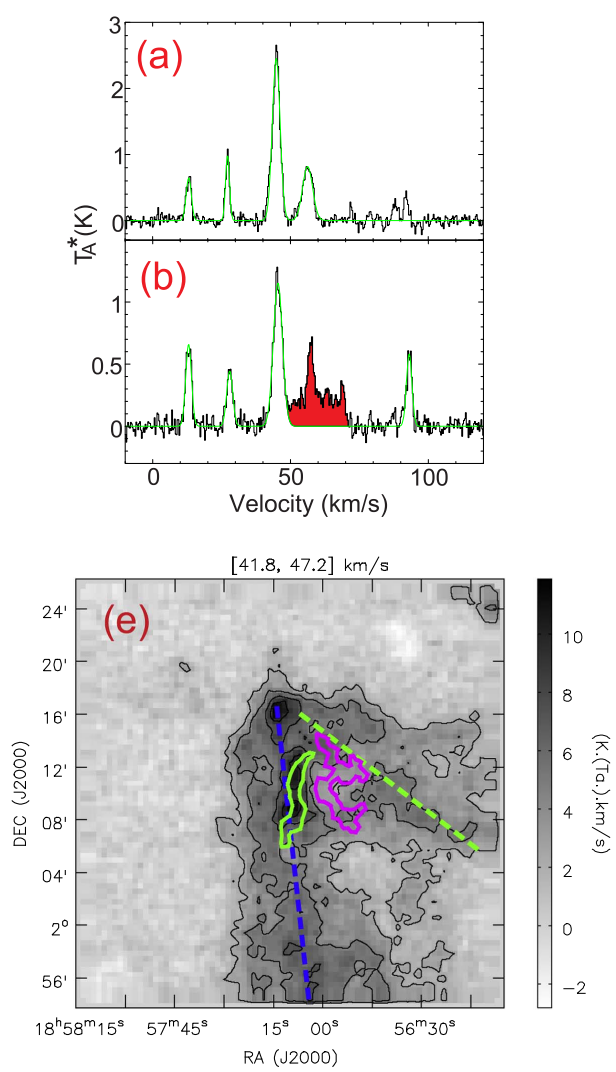
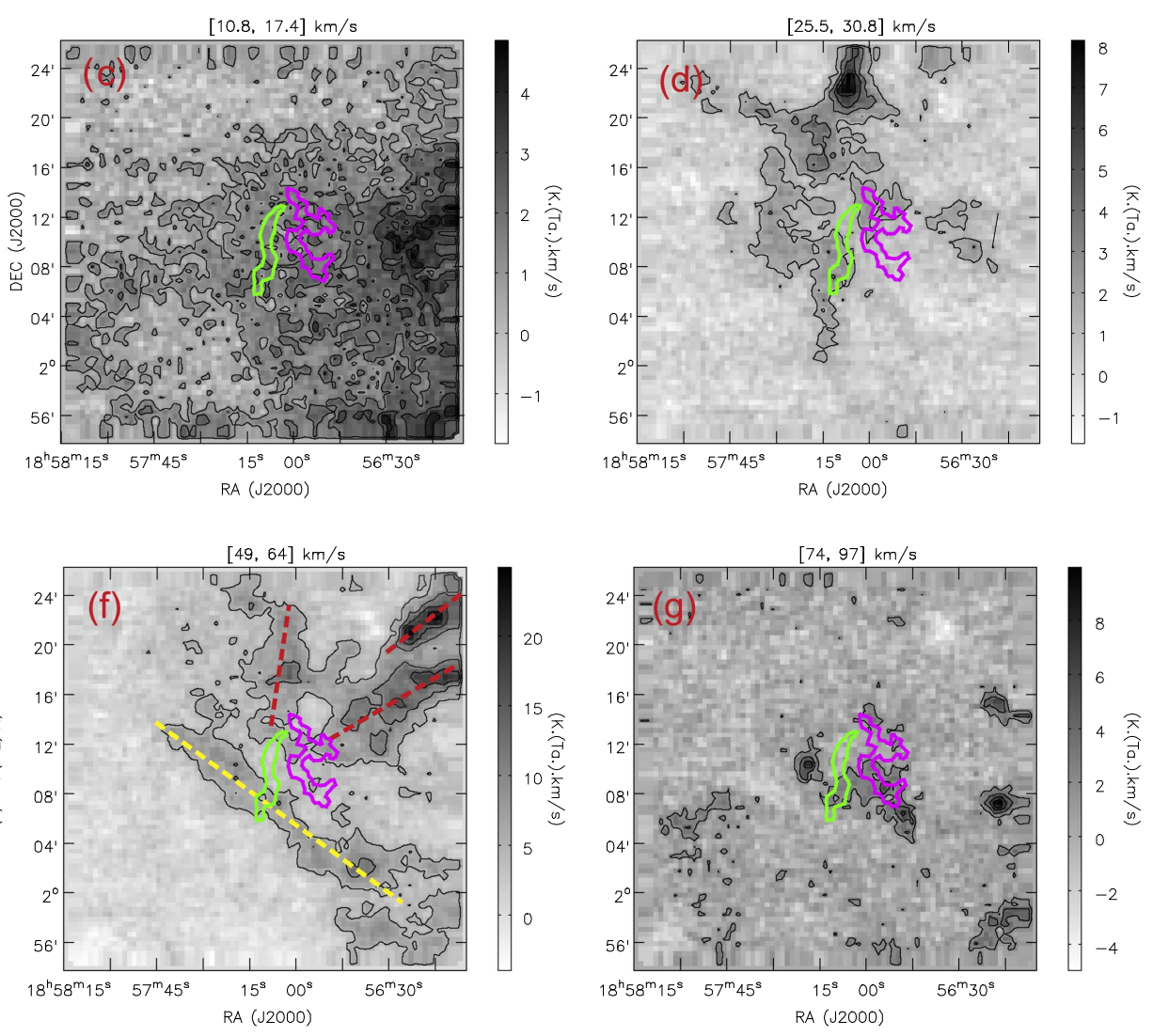

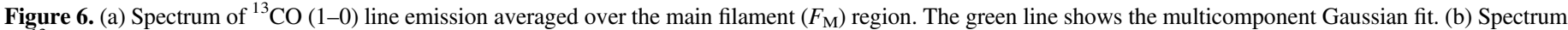

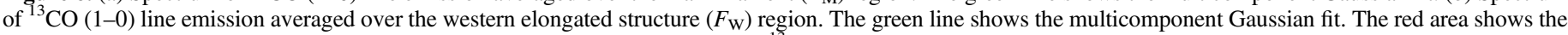

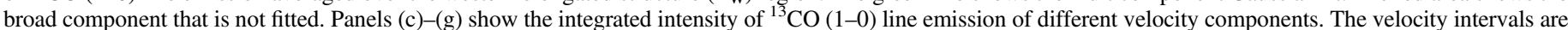

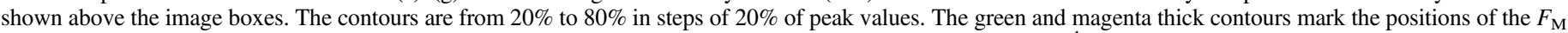

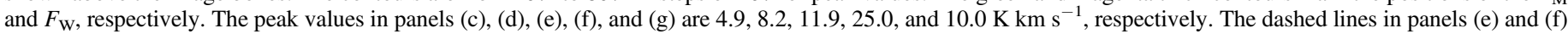
outline the elongated structures in the emission.

collision. $F_{\mathrm{M}}$ itself is also formed owing to the large-scale cloud-cloud collision.

From the channel maps of ${ }^{13} \mathrm{CO}(1-0)$ line emission (see the Appendix), we identify two velocity-coherent clouds (G035.39-main and G035.39-west) whose spatial distributions are distinctly different. Panel (a) of Figure 8 presents the moment 1 map of ${ }^{13} \mathrm{CO}(1-0)$ line emission toward the G035.39 clouds. Blueshifted emission (G035.39-main) and redshifted emission (G035.39-west) are clearly separated in the northern part of the map, indicating two clouds in collision. The southern part of G035.39-main is not affected by cloudcloud collision and thus shows no clear velocity gradient. Hernandez \& Tan (2015) argue that the large-scale velocity gradient in G035.39 is caused by cloud rotation. By inspecting the channel maps, however, the blueshifted emission gas and redshifted emission gas more likely belong to two different clouds. In addition, multiple velocity components in $F_{\mathrm{M}}$ have been seen in high spectral resolution observations (Henshaw et al. 2013), which cannot be well explained by the cloud rotation scenario.

The integrated intensity maps of ${ }^{13} \mathrm{CO}(1-0)$ for $\mathrm{G} 035.39$ west (46-47 $\mathrm{km} \mathrm{s}^{-1}$ ) and G035.39-main (41-43 $\mathrm{km} \mathrm{s}^{-1}$ ) are shown in panel (b) of Figure 8. In the colliding area, the cloud gas with redshifted velocities is well separated spatially from the cloud gas with blueshifted velocities. $F_{\mathrm{M}}$ is located in the interface layer, where the internal turbulence and the momentum exchange between the two colliding clouds may mix the gas distribution in both space and velocity and enhance the density therein. G035.39-west is curved as depicted by the yellow dashed line, suggesting that it is greatly compressed as it collides with G035.39-main. The widespread $\mathrm{SiO}$ emission in the northern part of $F_{\mathrm{M}}$ discovered by Jiménez-Serra et al. (2010) may be a sign of (large-scale) shocks from the resulting compression. The emission peak of G035.39-west is in the interface, suggesting that the majority of the gas of G035.39west may have merged with the gas of G035.39-main. G035.39-west seems to have swung during collision, forming a long tail in the west (as seen in the red dashed box). In addition, the northern ("N") part of $F_{\mathrm{M}}$ appears to be more affected by the collision than the middle and southern parts ("M" and "S") because the emission peak of G035.39-west is located close to the north end of $F_{\mathrm{M}}$. Moreover, the northern part of $F_{\mathrm{M}}$ is found to have more redshifted velocities than the southern part, as seen from the moment 1 map of ${ }^{13} \mathrm{CO}(1-0)$ line emission. We suspect that the collision occurs from the northwest of $F_{\mathrm{M}}$ and slows down as it propagates to the south, causing a velocity gradient along $F_{\mathrm{M}}$. Therefore, the previous findings of velocity gradients (Henshaw et al. 2014; JiménezSerra et al. 2014; Sokolov et al. 2017) and multiple velocity components (Henshaw et al. 2013) in $F_{M}$ can be explained by the mixed gas distribution from the two larger-scale colliding clouds.

The relative velocity between G035.39-west and G035.39main is $\sim 5 \mathrm{~km} \mathrm{~s}^{-1}$, which is similar to that of the two colliding filaments suggested by Henshaw et al. (2013). Considering the projection effect, the collision velocity of the two clouds could 
Table 2

Parameters of Molecular Lines

\begin{tabular}{|c|c|c|c|c|c|c|c|c|c|c|}
\hline Line & $\begin{array}{l}\text { Velocity } \\
\left(\mathrm{km} \mathrm{s}^{-1}\right)\end{array}$ & $\begin{array}{l}\text { FWHM } \\
\left(\mathrm{km} \mathrm{s}^{-1}\right)\end{array}$ & $\begin{array}{c}T_{b} \\
(\mathrm{~K})\end{array}$ & $\begin{array}{c}T_{\mathrm{d}} \\
(\mathrm{K})\end{array}$ & $\begin{array}{c}N_{\mathrm{H} 2} \\
\left(\mathrm{~cm}^{-2}\right)\end{array}$ & $\begin{array}{c}n \\
\left(\mathrm{~cm}^{-3}\right)\end{array}$ & $\begin{array}{c}N_{\text {line }} \\
\left(\mathrm{cm}^{-2}\right)\end{array}$ & $X_{\text {line }}$ & $\tau$ & $\begin{array}{l}T_{\mathrm{ex}} \\
(\mathrm{K})\end{array}$ \\
\hline \multicolumn{11}{|c|}{$F_{\mathrm{M}}$ within $100 \mathrm{mJy}$ beam $^{-1}$ Contour of $850 \mu \mathrm{m}$ Continuum } \\
\hline \multirow[t]{4}{*}{${ }^{13} \mathrm{CO}(1-0)$} & $44.9 \pm 0.1$ & $3.3 \pm 0.1$ & $4.9 \pm 0.1$ & $14 \pm 1$ & $2.4 \times 10^{22}$ & $1.0 \times 10^{4}$ & $2.2 \times 10^{16}$ & $9.2 \times 10^{-7}$ & 0.6 & 14.1 \\
\hline & $13.1 \pm 0.1$ & $2.2 \pm 0.2$ & $1.3 \pm 0.1$ & & & & & & & \\
\hline & $27.2 \pm 0.1$ & $1.7 \pm 0.1$ & $2.0 \pm 0.1$ & & & & & & & \\
\hline & $56.2 \pm 0.1$ & $4.3 \pm 0.2$ & $1.6 \pm 0.1$ & & & & & & & \\
\hline $\mathrm{C}^{18} \mathrm{O}(1-0)$ & $44.9 \pm 0.1$ & $2.1 \pm 0.1$ & $1.2 \pm 0.1$ & $14 \pm 1$ & $2.4 \times 10^{22}$ & $1.0 \times 10^{4}$ & $2.6 \times 10^{15}$ & $1.1 \times 10^{-7}$ & 0.1 & 15.0 \\
\hline \multicolumn{11}{|c|}{$F_{\mathrm{W}}$ within $10 \mathrm{mJy}$ beam $^{-1}$ Contour of $850 \mu \mathrm{m}$ Continuum } \\
\hline \multirow[t]{4}{*}{${ }^{13} \mathrm{CO}(1-0)$} & $45.4 \pm 0.1$ & $3.9 \pm 0.3$ & $2.4 \pm 0.1$ & $21 \pm 1$ & $4.0 \times 10^{21}$ & $1.7 \times 10^{3}$ & $8.5 \times 10^{15}$ & $2.1 \times 10^{-6}$ & 0.2 & 14.5 \\
\hline & $13.0 \pm 0.1$ & $2.5 \pm 0.3$ & $1.3 \pm 0.1$ & & & & & & & \\
\hline & $27.9 \pm 0.2$ & $2.6 \pm 0.5$ & $0.9 \pm 0.1$ & & & & & & & \\
\hline & $93.1 \pm 0.2$ & $2.3 \pm 0.4$ & $1.1 \pm 0.1$ & & & & & & & \\
\hline $\mathrm{C}^{18} \mathrm{O}(1-0)$ & $44.9 \pm 0.1$ & $1.8 \pm 0.4$ & $0.3 \pm 0.1$ & $21 \pm 1$ & $4.0 \times 10^{21}$ & $1.7 \times 10^{3}$ & $4.5 \times 10^{14}$ & $1.1 \times 10^{-7}$ & 0.03 & 14.3 \\
\hline
\end{tabular}

be $\sim 10 \mathrm{~km} \mathrm{~s}^{-1}$ (for an inclination angle of $45^{\circ}$ ), consistent with the collision speeds of GMCs in some simulations (Inoue \& Fukui 2013; Wu et al. 2015, 2017). A cloud-cloud collision in G035.39 is also supported by [C II] observations (Bisbas et al. 2018).

A schematic illustration of the cloud-cloud collision is shown in panels (a) and (b) of Figure 9. The smaller G035.39west cloud collides with the northern part of G035.39-main in a northwest-southeast direction. The cloud-cloud collision enhances the density in the interface, where the massive filament $F_{\mathrm{M}}$ has formed. The dynamical effect of the cloudcloud collision may perturb $F_{\mathrm{M}}$ and trigger its fragmentation. In contrast, the southern part (blue dashed box in panel (b) of Figure 8) of G035.39-main is not affected by the cloud-cloud collision, and its density is not enhanced, as seen from our ${ }^{13} \mathrm{CO}$ map, as well as infrared extinction maps (Kainulainen \& Tan 2013). No dense clump (or new stars) has been formed there yet. Therefore, cloud-cloud collision can enhance density and shorten the local freefall timescale for star formation.

\subsection{The Origin of Magnetic Field Geometry Surrounding the Main Filament}

As discussed in Section 3.1, the magnetic field orientations are roughly perpendicular to the major axis of $F_{\mathrm{M}}$ along the central ridge and at the junctions with other filaments, while magnetic field orientations tend to be oblique in the lowerdensity surroundings (see panel (d) in Figure 3). To explore the large-scale field geometry further, we convolve the Stokes $Q$, $U$, and $I$ maps of POL- 2 data with a $2^{\prime}$ beam and recalculate the polarization angles from the smoothed maps. The smoothed magnetic field orientations are overlaid onto the similar Stokes I image in Figure 10. The smoothed magnetic field orientations may indicate that the magnetic field is pinched around the middle and southern parts (marked by the blue dashed ellipse in Figure 10) of $F_{\mathrm{M}}$. The pinched magnetic field can be associated with the accretion flow around and along the filament in a globally collapsing cloud (Klassen et al. 2017; Gomez et al. 2018; P. S. Li et al. 2018, in preparation). Therefore, the magnetic fields in these regions seem to have been dragged by the collapsing gas flow that forms those dense structures ( $\mathrm{Li}$ et al. 2015b, 2018, in preparation; Klassen et al. 2017; Gomez et al. 2018).
As shown in Figure 1 in Gomez et al. (2018), the magnetic field lines can be dragged by the accretion flow. In low-density regions away from a filament, the gas flow direction is perpendicular to the filament, and the dragged magnetic field must be mainly perpendicular to the filament as seen in the surroundings of other filamentary clouds (Chapman et al. 2011; Cox et al. 2016; Santos et al. 2016). In the low-density regions surrounding the filament spine, the magnetic field is affected by accretion flows both onto and along the filament, and thus the magnetic field lines must also develop a component parallel to the filament and appear oblique. Accretion flows along the filament can compress the magnetic field at the filament spine and increase the perpendicular component as seen in simulations. This picture, illustrated in panel (c) of Figure 9, can well explain the magnetic field geometry in the middle part of $F_{\mathrm{M}}$, where the projected magnetic field lines are mainly perpendicular to the filament along its densest regions and are oblique in less dense regions (see panel (d) in Figure 3).

As shown in panel (b) of Figure 3, the magnetic field orientations in the northern end of $F_{\mathrm{M}}$ are nearly parallel to the filament. This pattern cannot be explained by gas flows along the dense filament because such flows would not increase the parallel component of the magnetic field, but would rather increase the perpendicular component, leading to a "U"-shaped magnetic geometry (Gomez et al. 2018; P. S. Li et al. 2018, in preparation). As marked by the green dashed box in Figure 10, the smoothed magnetic field orientations in a large area close to the northern end of the $F_{\mathrm{M}}$ are well aligned along the northwest-southeast direction. Indeed, such a nearly parallel pattern may be due to compression from the east (see panel (c) in Figure 9). Since the northern part of $F_{\mathrm{M}}$ is more affected by the cloud-cloud collision (see Section 4.1), the northern end of $F_{\mathrm{M}}$ may be elongated and compressed by the two colliding clouds. The magnetic field at this location is well aligned with the elongated filament (see Figure 9(c)).

\subsubsection{Pinched Magnetic Field Surrounding Clump "c8”}

The magnetic field surrounding clump "c8" is shown in panel (a) of Figure 11. The magnetic field orientations associated with "c8" are likely pinched. The pinched magnetic field may hint at gas inflows toward the center of "c8." The yellow dashed arrows in the panel indicate the possible gas flow directions. Interestingly, the magnetic field orientations 

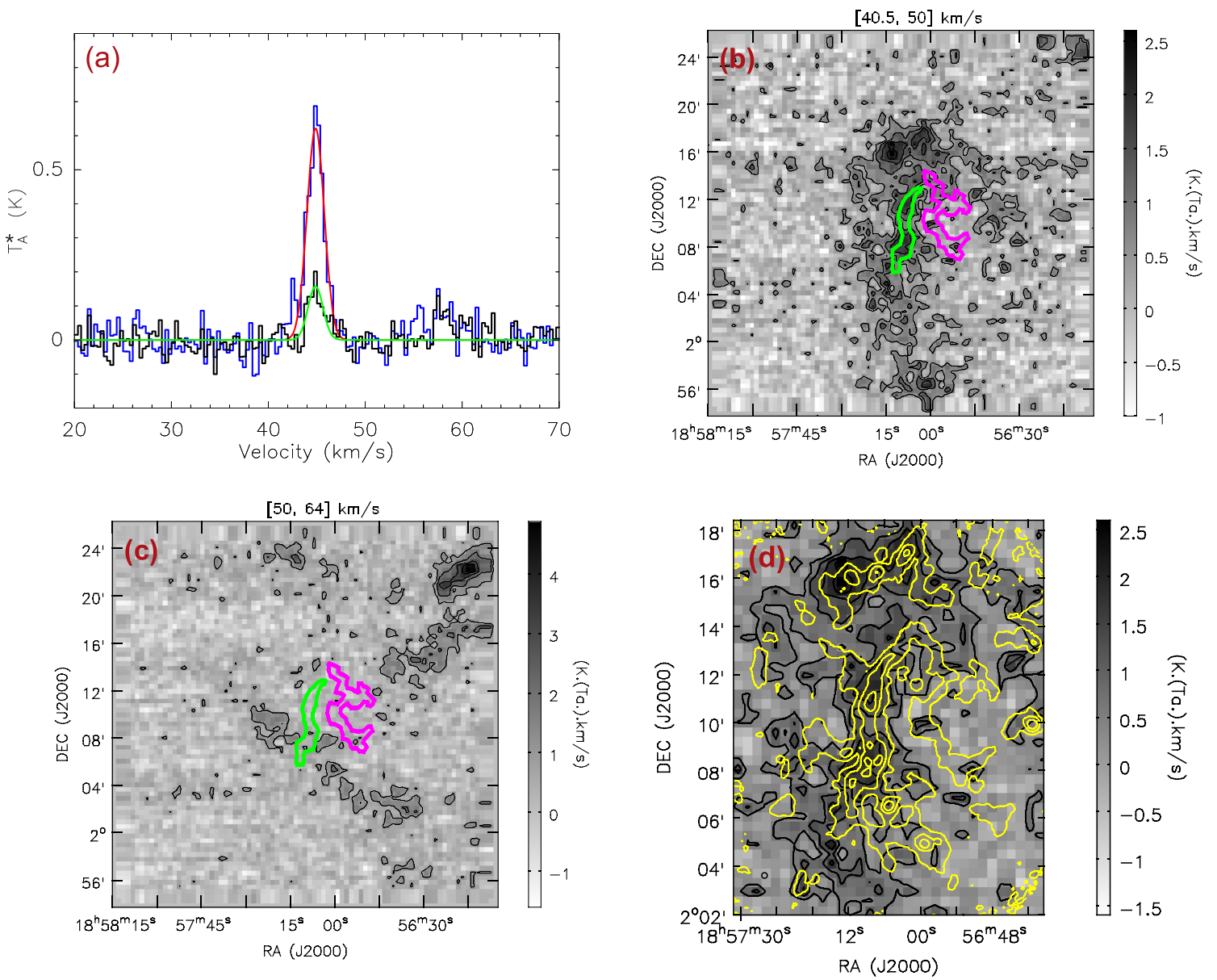

Figure 7. (a) Spectra of $\mathrm{C}^{18} \mathrm{O}(1-0)$ line emission averaged over $F_{\mathrm{M}}$ and $F_{\mathrm{W}}$ are shown in blue and black, respectively. The red and green curves show Gaussian fits. Panels (b) and (c) show the integrated intensity of $\mathrm{C}^{18} \mathrm{O}(1-0)$ line emission of different velocity components. The velocity intervals are shown above the image boxes. The contours are from $20 \%$ to $80 \%$ in steps of $20 \%$ of peak values. The peak values in panels (b) and (c) are 2.6 and $4.9 \mathrm{~K} \mathrm{~km} \mathrm{~s}^{-1}$, respectively. The green and magenta thick contours mark the positions of $F_{\mathrm{M}}$ and $F_{\mathrm{W}}$, respectively. (d) The gray image and black contours show the integrated intensity of $\mathrm{C}^{18} \mathrm{O}(1-0)$ line emission as the same in panel (b). The yellow contours show $850 \mu \mathrm{m}$ continuum emission. The contours are $[0.15,0.5,1,2,3,4,5,6] \times 100 \mathrm{mJy}^{2}$ beam ${ }^{-1}$.

are roughly parallel to the suggested gas flow directions, a behavior seen also in simulations (Klassen et al. 2017; Gomez et al. 2018; P. S. Li et al. 2018, in preparation). In addition, the magnetic field orientations close to the center become more perpendicular to the major axis of the local filament, suggesting that magnetic fields therein have been compressed by accretion flows and become more perpendicular as a result (Klassen et al. 2017; Gomez et al. 2018; P. S. Li et al. 2018, in preparation). Indeed, the magnetic fields surrounding "c8" hint at a "U"shaped geometry caused by accretion flows (Gomez et al. 2018; P. S. Li et al. 2018, in preparation). The coarse resolution of our POL-2 observations, however, cannot resolve the field geometry of the clumps. In addition, there are only a handful of high-S/N orientations, too few to well constrain the field geometry. Future higher-sensitivity and higher-resolution polarization observations are needed to investigate the field geometry in greater detail.

In panel (b) of Figure 11, we show the magnetic field surrounding an accretion core from a simulation of an IRDC (Li et al. 2015b, 2018, in preparation). This image is taken from an ideal MHD turbulence simulation driven at a thermal Mach number of 10 and an Alfvén Mach number of 1. The image is selected at half of the freefall time of the simulation. The core (bright yellow region) is accreting gas along the filament. The white dashed arrow shows the accretion direction. The magnetic field has been twisted along the accretion direction. Close to the densest part of the core, the magnetic field is compressed and is roughly perpendicular to the filament. The accretion significantly increases the perpendicular component of the magnetic field at the filament spine. Although " $\mathrm{c} 8$ " is much more massive and larger than the simulated core, the magnetic field surrounding " $\mathrm{c} 8$ " shows similar geometry to that associated with the simulated core, suggesting that the magnetic field surrounding " $\mathrm{c} 8$ " has been similarly pinched by gas inflow along the filament.

Using ALMA, Henshaw et al. (2017) resolved clump "c3" into a network of narrow $(\sim 0.028 \pm 0.005 \mathrm{pc}$ in width $)$ subfilaments that contain 28 compact cores. Those cores may be still accreting gas along those subfilaments. Therefore, we suspect that the magnetic fields surrounding those cores are also pinched owing to the accretion, as seen in simulations (see panel (b) of Figure 11). Future polarization observations with ALMA will shed light on the magnetic field geometry surrounding these cores.

\subsection{Gravitational Stability of the Main Filament}

Most stability analyses of massive filaments suggest that the magnetic field is important, but a thorough analysis has been elusive given the difficulties in observing magnetic fields (e.g., Jackson et al. 2010; Contreras et al. 2016; Henshaw et al. 2016; 

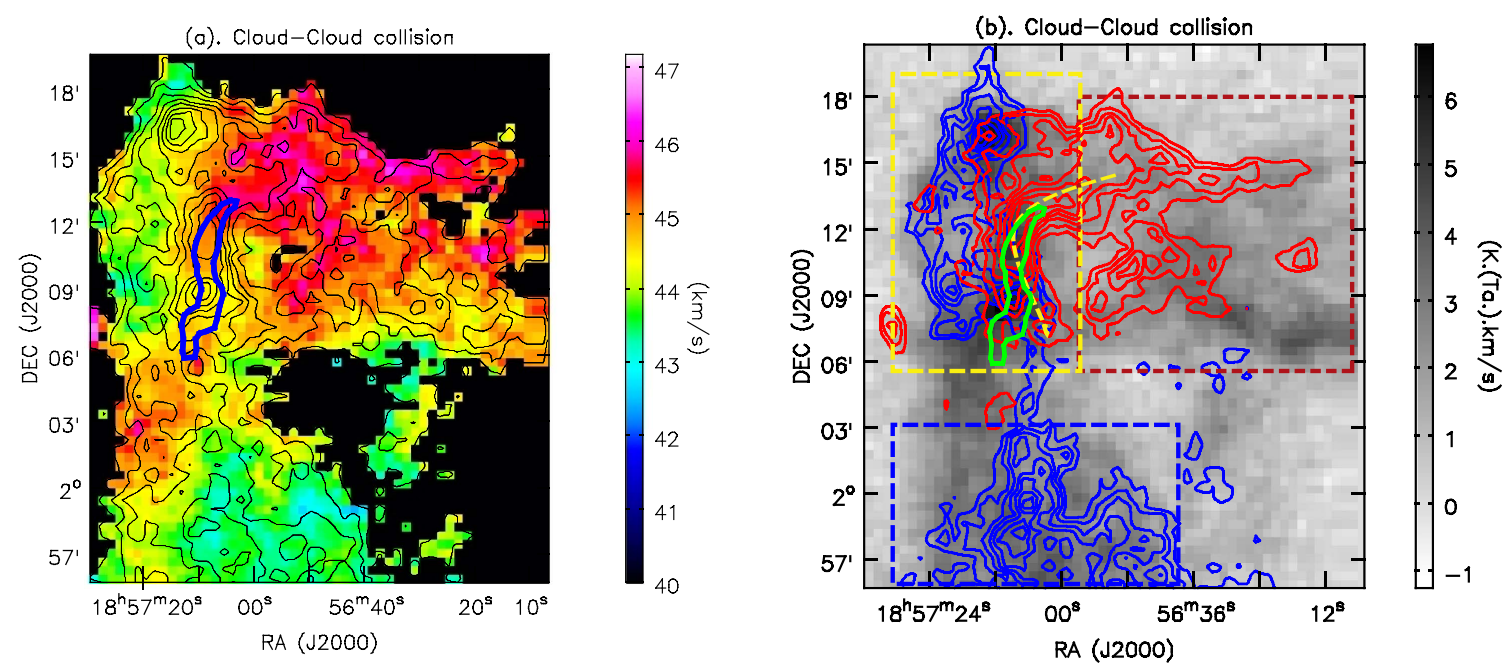

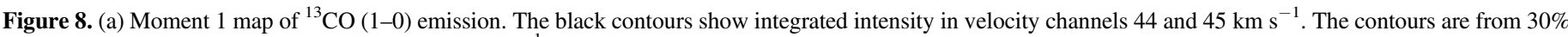

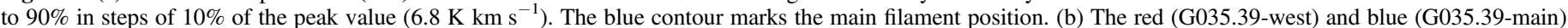

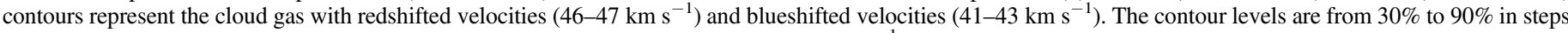

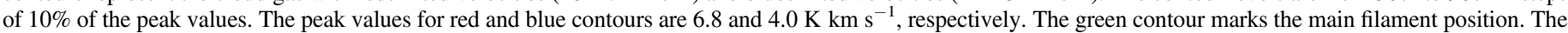

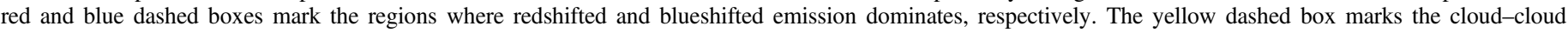
collision area. The yellow dashed curve roughly shows the shell-like structure in the redshifted gas emission.

Lu et al. 2018). In this section, we investigate the gravitational stability of the main filament $F_{\mathrm{M}}$ by taking into account thermal pressure, turbulence, and magnetic fields.

The critical line mass for the global gravitational stability of an isothermal filament supported by thermal pressure and turbulence is (Ostriker 1964; Pattle et al. 2017)

$$
\left(\frac{M}{L}\right)_{\text {crit }}=\frac{2 \sigma_{3 \mathrm{D}}^{2}}{G}
$$

where $G$ is the gravitational constant. Assuming that the velocity dispersion is isotropic, the three-dimensional velocity dispersion is

$$
\sigma_{3 \mathrm{D}}=\sqrt{3\left(\sigma_{\mathrm{NT}}^{2}+c_{s}^{2}\right)} .
$$

We obtain a mean nonthermal velocity dispersion $\left(\sigma_{\mathrm{NT}}\right)$ of $\sim 0.4 \mathrm{~km} \mathrm{~s}^{-1}$ from the line widths of the $\mathrm{NH}_{3}(1,1)$ line (Sokolov et al. 2017). The 1D thermal velocity dispersion (or sound speed) is

$$
c_{s}=\sqrt{\frac{k T_{k}}{\mu m_{\mathrm{H}}}}
$$

where $\mu=2.37$ is the mean molecular weight per "free particle" $\left(\mathrm{H}_{2}\right.$ and $\mathrm{He}$; the number of metal particles is negligible). $c_{s}$ is $\sim 0.23 \mathrm{~km} \mathrm{~s}^{-1}$ for a mean kinetic temperature $\left(T_{\mathrm{k}}\right)$ of $15 \mathrm{~K}$. Here we assume that $T_{\mathrm{k}}$ equals $T_{\mathrm{d}}$ under the local thermodynamic equilibrium (LTE) conditions. Therefore, the $\sigma_{3 \mathrm{D}}$ and $(M / L)_{\text {crit }}$ are $0.80 \mathrm{~km} \mathrm{~s}^{-1}$ and $\sim 296 M_{\odot} \mathrm{pc}^{-1}$, respectively. The $(M / L)_{\text {crit }}$ is smaller than the measured line mass $\left(\sim 410 M_{\odot} \mathrm{pc}^{-1}\right)$, suggesting that the filament cannot be supported against collapse only by turbulent gas pressure in the absence of magnetic fields.

In contrast, the $F_{\mathrm{W}}$ filament is as turbulent as the $F_{\mathrm{M}}$ but is much less dense. The critical line mass for $F_{\mathrm{W}}$ is comparable to that of $F_{\mathrm{M}}$, while its line mass is only $\sim 100 M_{\odot} \mathrm{pc}^{-1}$. Therefore, turbulent motions in $F_{\mathrm{W}}$ can dominate over gravity in $F_{\mathrm{M}}$ and may further stretch $F_{\mathrm{W}}$.
The criterion for filament stability with support from magnetic fields can be estimated as (Ostriker 1964; Fiege \& Pudritz 2000; Pattle et al. 2017)

$$
\left(\frac{M}{L}\right)_{\text {crit,mag }}=\left(\frac{M}{L}\right)_{\text {crit }}\left(1-\frac{\mathcal{M}_{\mathcal{B}}}{|\mathcal{W}|}\right)^{-1},
$$

where $\mathcal{M}_{\mathcal{B}}$ is the magnetic energy per unit length and $|\mathcal{W}|=\left(\frac{M}{L}\right) G \approx 4.6 \times 10^{26} \mathrm{erg} \mathrm{cm}^{-1}$ is the gravitational energy per unit length. $\mathcal{M}_{\mathcal{B}}$ may be either positive or negative, depending on whether a poloidal or toroidal field, respectively, dominates the overall magnetic energy (Fiege \& Pudritz 2000). If a poloidal field dominates, the factor $\left(1-\frac{\mathcal{M}_{\mathcal{B}}}{|\mathcal{W}|}\right)^{-1}$ is larger than 1 (Fiege \& Pudritz 2000). Therefore, a poloidal field helps to support the cloud radially against self-gravity by increasing the critical mass per unit length (Fiege \& Pudritz 2000). In contrast, the factor $\left(1-\frac{\mathcal{M}_{\mathcal{B}}}{|\mathcal{W}|}\right)^{-1}$ is smaller than 1 (Fiege \& Pudritz 2000) for a toroidal field. A toroidal field works with gravity in squeezing the cloud by reducing the critical mass per unit length (Fiege \& Pudritz 2000).

The total magnetic field strength $\left(B_{\mathrm{tot}}\right)$ can be estimated using the Davis-Chandrasekhar-Fermi (DCF) method (Davis \& Greenstein 1951; Chandrasekhar \& Fermi 1953):

$$
B_{\mathrm{tot}}=1.3 B_{\mathrm{pos}}=1.3 Q^{\prime} \sqrt{4 \pi \rho} \frac{\sigma_{\mathrm{NT}}}{\sigma_{\theta}},
$$

where $B_{\text {pos }}$ is the POS magnetic field strength, 1.3 is a factor considering projection effects, $Q^{\prime}$ is a factor of order unity accounting for variations in field strength on scales smaller than the beam (Crutcher et al. 2004), $\rho=\mu_{g} m_{\mathrm{H}} n_{\mathrm{H}_{2}}$ is the gas density, and $\sigma_{\theta}$ is the dispersion in polarization position angles. Here $Q^{\prime}$ is taken as 0.5 (Ostriker et al. 2001).

As seen in Figure 3, the magnetic field orientations in the middle part of $F_{\mathrm{M}}$ are well ordered and uniform, with their orientations roughly perpendicular to the major axis of the filament. In addition, the magnetic field orientations tend to be 


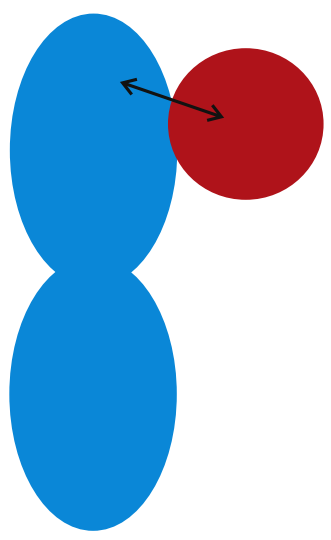

(a)

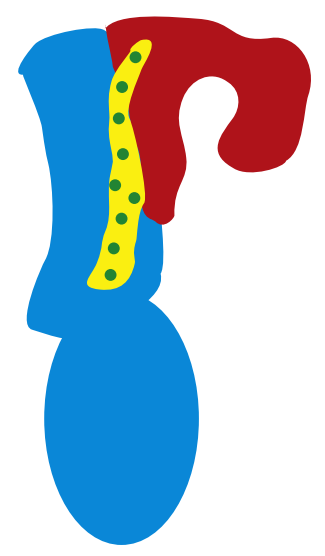

(b)

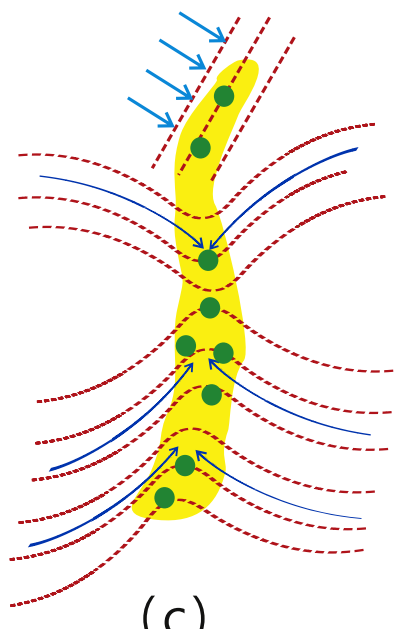

(c)

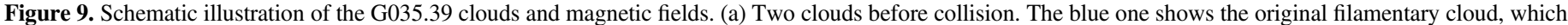

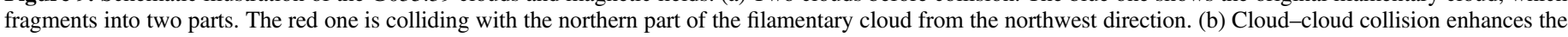

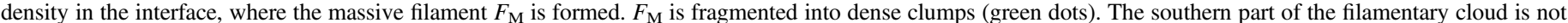

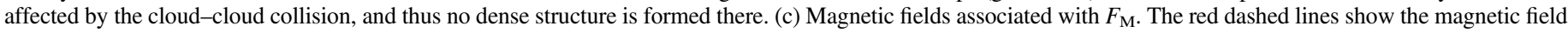

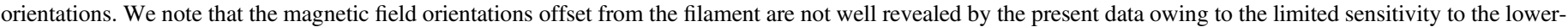
density gas polarization signal. The blue arrows show the gas flow direction.

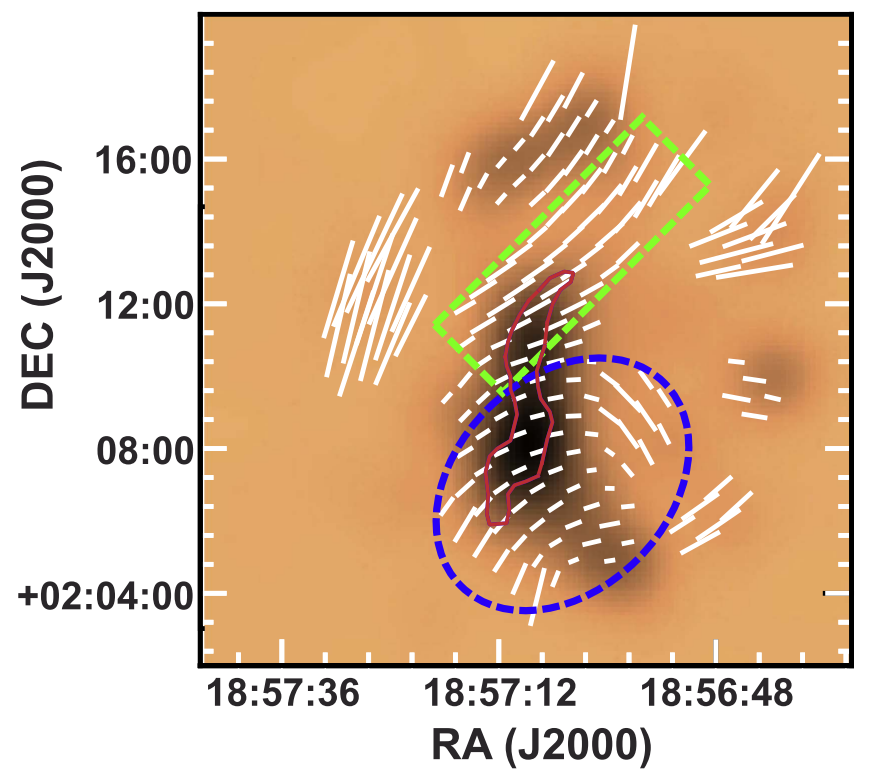

Figure 10. Smoothed JCMT/POL-2 map of G035.39. The $Q, U$, and $I$ maps of G035.39 were smoothed with a beam size of $2^{\prime}$. The white orientations represent the smoothed magnetic field orientations. The red contour outlines the massive filament $F_{\mathrm{M}}$. The green box and blue ellipse mark the regions showing different magnetic field geometry.

more perpendicular toward the denser regions (see panel (d) of Figure 3). In contrast, the magnetic field orientations in the northern and southern ends are more widely dispersed in direction. We only estimated $\sigma_{\theta}$ in the middle part of the main filament, where the Stokes $I$ intensity at $850 \mu \mathrm{m}$ is above $100 \mathrm{mJy}$ beam $^{-1}$. As shown in panel (a) of Figure 4, from a Gaussian fit to the orientation angles, the measured $\sigma_{\theta}$ is $\sim 17^{\circ}$. We correct the angular dispersion $\sigma_{\theta}$ by subtracting the measurement uncertainty $\left(\delta_{\theta} \sim 9^{\circ}\right)$ with $\sqrt{\sigma_{\theta}^{2}-\delta_{\theta}^{2}}$. The corrected $\sigma_{\theta}$ is $\sim 15^{\circ}$, which is smaller than the maximum value at which the standard DCF method can be safely applied ( $\leqslant 25^{\circ}$; Heitsch et al. 2001). Taking $\sigma_{\theta}$ as $\sim 15^{\circ}$, we obtain a
POS magnetic field strength ( $\left.B_{\text {pos }}\right)$ of $50 \mu \mathrm{G}$ and hence a total magnetic field strength $\left(B_{\text {tot }}\right)$ of $65 \mu \mathrm{G}$.

The estimated magnetic field strength should be treated as an upper limit because (1) $\sigma_{\theta}$ is estimated from the densest region of $F_{\mathrm{M}}$ with uniform magnetic field orientations. The magnetic field orientations in other parts of $F_{\mathrm{M}}$ are more widely dispersed and should have larger $\sigma_{\theta}$. Therefore, $\sigma_{\theta}$ used in the above calculations with the DCF method is a lower limit. (2) $\sigma_{\mathrm{NT}}$ is estimated from the mean line width of $\mathrm{NH}_{3}(1,1)$ (Sokolov et al. 2017). The GBT beam at $\mathrm{NH}_{3}(1,1)$ line frequency is $32^{\prime \prime}$, larger than the 14 !" 1 beam of SCUBA-2/ POL-2 at $850 \mu \mathrm{m}$. Therefore, some uncertainties remain in $\sigma_{\mathrm{NT}}$ for the estimation of magnetic field strength using the DCF method at this scale.

Crutcher et al. (2010) obtained an empirical relation for a maximum field strength $\left(B_{\max }\right)$ versus density from Zeeman observations:

$$
B_{\max } \simeq 0.22\left(\frac{n_{\mathrm{H}}}{10^{5} \mathrm{~cm}^{-3}}\right)^{0.65} \mathrm{mG}\left(n_{\mathrm{H}}>300 \mathrm{~cm}^{-3}\right) .
$$

For a mean $n_{\mathrm{H}}$ of $\sim 1.5 \times 10^{4} \mathrm{~cm}^{-3}$ in $F_{\mathrm{M}}$, the maximum field strength $B_{\max }$ estimated from the empirical relation is $64 \mu \mathrm{G}$, i.e., similar to the value $(65 \mu \mathrm{G})$ derived from the DCF method. Therefore, $65 \mu \mathrm{G}$ may represent an upper limit for the mean magnetic field strength of the main filament $F_{\mathrm{M}}$.

Although lacking an accurate determination of magnetic field strength, we can use the upper limit of $65 \mu \mathrm{G}$ to evaluate the importance of magnetic field in the gravitational stability of the main filament.

The corresponding Alfvénic velocity for a magnetic field strength of $65 \mu \mathrm{G}$ is

$$
\sigma_{A}=\frac{B_{\mathrm{tot}}}{\sqrt{4 \pi \rho}}
$$

where $\sigma_{A} \approx 1.0 \mathrm{~km} \mathrm{~s}^{-1}$ for $B_{\text {tot }}=65 \mu \mathrm{G}$ and a mean volume density of $7.3 \times 10^{3} \mathrm{~cm}^{-3}$. The Alfvén Mach number is

$$
\mathcal{M}_{\mathcal{A}}=\sqrt{3} \sigma_{\mathrm{NT}} / \sigma_{A}
$$




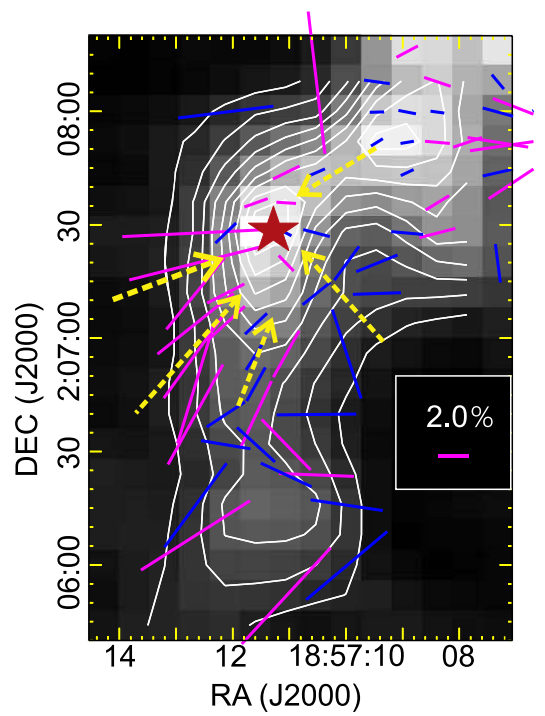

(a). POL-2 map of clump "c8"

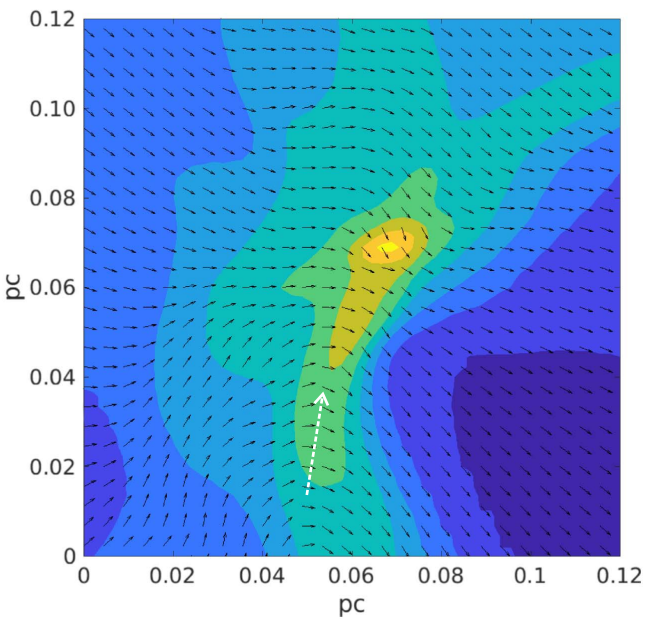

(b). Magnetic field streamlines surrounding an accreting core in simulations

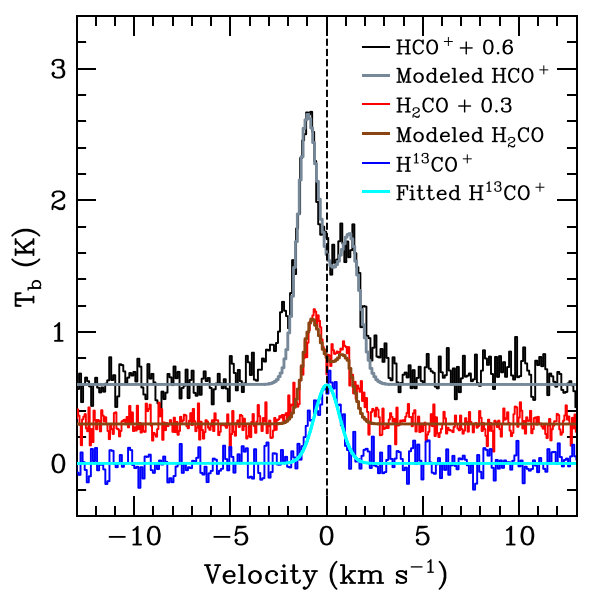

(c). Spectra at the central position of "c8"

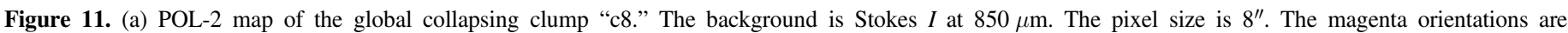

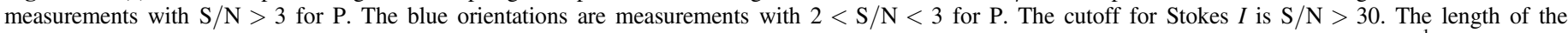

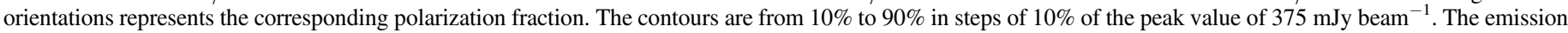

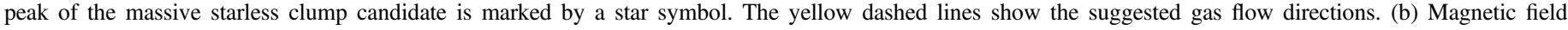

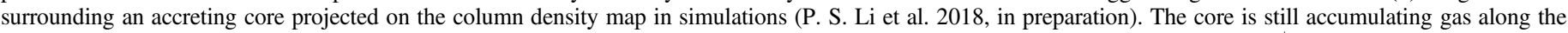

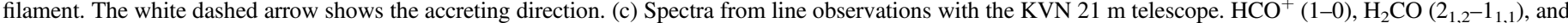

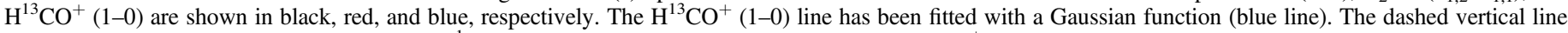

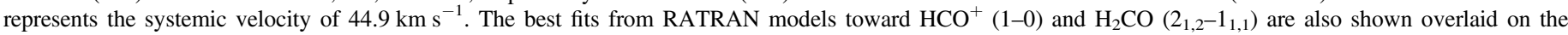
observed spectra.

We derived $\mathcal{M}_{\mathcal{A}} \approx 0.7$, suggesting that the turbulent motions may be sub-Alfvénic in the main filament. The total magnetic energy $\left(E_{B}\right)$ is (Pattle et al. 2017)

$$
E_{B}=\frac{B_{\mathrm{tot}}^{2} V}{2 \mu_{0}}
$$

where $\mu_{0}$ is the permeability of free space. Therefore, the magnetic energy per unit length is

$$
\left|\mathcal{M}_{\mathcal{B}}\right|=\frac{E_{B}}{L}
$$

Considering the volume $\left(V=5.4 \mathrm{pc}^{3}\right)$ and length $(L=6.8 \mathrm{pc})$ of $F_{\mathrm{M}}, E_{B}$ and $\left|\mathcal{M}_{\mathcal{B}}\right|$ are $\sim 2.7 \times 10^{46}$ erg and $\sim 1.3 \times$ $10^{26} \mathrm{erg} \mathrm{cm}^{-1}$, respectively. Therefore, if a poloidal field component dominates, it will increase the critical line mass by a factor of $\left(1-\frac{1.3 \times 10^{26}}{4.6 \times 10^{26}}\right)^{-1} \sim 1.39$. In this case, the critical line mass, taking into account the additional support from magnetic fields, will become $\sim 411 M_{\odot} \mathrm{pc}^{-1}$, which is very similar to the measured value $\left(\sim 410 M_{\odot} \mathrm{pc}^{-1}\right)$. If, however, a toroidal field component dominates, it will decrease the critical line mass by a factor of $\left(1-\frac{-1.3 \times 10^{26}}{4.6 \times 10^{26}}\right)^{-1} \sim 0.78$. If so, the critical line mass will become $\sim 231 M_{\odot} \mathrm{pc}^{-1}$, much smaller than the measured value $\left(\sim 410 M_{\odot} \mathrm{pc}^{-1}\right)$.

Judging from panels (a) and (b) in Figure 3, the northern part ("N") of $F_{\mathrm{M}}$ has magnetic field orientations parallel to the major axis, indicating that the magnetic field therein is likely poloidal. Therefore, the northern part may be stable with additional support from magnetic fields.

In contrast, the middle part of $F_{\mathrm{M}}$ is dominated by a magnetic field whose orientation is perpendicular to the major axis, resembling the projection of a toroidal magnetic field wrapping around the filament. If so, the middle part may become more unstable. Alternatively, the field can also just simply go straight through the filament, providing no support against gravitational collapse. Therefore, the middle part is likely unstable and may further fragment or collapse. Indeed, clump "c3" in the middle part already contains plenty of substructures (subfilaments and cores) detected in ALMA observations (Henshaw et al. 2017).

The southern part of $F_{\mathrm{M}}$ is more complicated. In contrast to the middle part, the magnetic field orientations in the southern part are more parallel to the major axis. We note, however, that the magnetic field orientations tend to be more perpendicular to the major axis in the densest region of the southern part. Therefore, the magnetic field in the southern part may contain comparable toroidal and poloidal components. The critical line mass considering magnetic field support will not deviate too much from that without magnetic field support. Hence, the southern part may be unstable and may fragment or collapse.

\subsection{Gravitational Stability of Dense Clumps}

In this section, we investigate the gravitational stability of dense clumps from virial analysis by taking into account the support from thermal pressure, turbulence, and magnetic fields.

If we only consider support from thermal pressure and turbulence, the virial masses $\left(M_{\mathrm{vir}}\right)$ of the clumps, assuming a uniform density profile, are (Bertoldi \& McKee 1992; Pillai et al. 2011; Sanhueza et al. 2017)

$$
M_{\mathrm{vir}}=\frac{5 R_{\mathrm{eff}}}{G}\left(\sigma_{\mathrm{NT}}^{2}+c_{s}^{2}\right) .
$$


The virial masses calculated are presented in Table 1. Three clumps ("c1," "c2," and "c5") have virial masses that are two to three times larger than their clump masses and hence may be gravitationally unbound, suggesting that "turbulent" gas motions in the clumps provide enough support against selfgravity. The other clumps have virial masses smaller than their clump masses, suggesting that they are bound and unstable without additional support from magnetic fields.

Henshaw et al. (2016) also suggested that the dense cores revealed in high-resolution interferometric observations are susceptible to gravitational collapse without additional support from magnetic fields. Therefore, it is important to evaluate the importance of magnetic fields in the gravitational stability of the dense clumps. Our SCUBA-2/POL-2 observations, however, do not resolve the magnetic fields surrounding those clumps, and thus we do not have estimation of their magnetic field strengths from observations. Instead, we estimate the magnetic field strengths with the empirical relation from Crutcher et al. (2010) and Li et al. (2015b).

Based on their MHD simulation results, Li et al. (2015b) suggested that the average field strength $\left(B_{\text {clump }}\right)$ in molecular clumps in the interstellar medium is

$$
B_{\text {clump }} \simeq 42\left(\frac{n_{\mathrm{H}}}{10^{4} \mathrm{~cm}^{-3}}\right)^{0.65} \mu \mathrm{G} .
$$

Using Equation (18), we estimated the total magnetic field strength $B_{\text {clump }}$ for clumps. The $B_{\text {clump }}$ and Alfvénic speed $\sigma_{A}$ of clumps are listed in Table 1 . The $B_{\text {clump values range from }}$ $\sim 56$ to $219 \mu \mathrm{G}$. The mean Alfvén Mach number of clumps is $\sim 0.75$, suggesting that the magnetic field may play a role as important as turbulence in supporting clumps against gravity. To investigate the gravitational stability of those dense clumps, we estimated the virial masses $\left(M_{\mathrm{vir}}^{B}\right)$ of the clumps considering thermal, turbulent, and magnetic pressures and assuming a uniform density profile (Bertoldi \& McKee 1992; Pillai et al. 2011; Sanhueza et al. 2017):

$$
M_{\mathrm{vir}}^{B}=\frac{5 R_{\mathrm{eff}}}{G}\left(\sigma_{\mathrm{NT}}^{2}+C_{s}^{2}+\frac{\sigma_{A}^{2}}{6}\right) .
$$

$M_{\text {vir }}^{B}$ are also presented in Table 1. Three clumps ("c1," "c2," and "c5") have virial masses two to three times larger than their clump masses and hence may be gravitationally unbound, suggesting that "turbulent" gas motions and magnetic fields in them provide significant support against self-gravity. The most two massive clumps ("c3" and "c8"), however, have clump masses larger than its virial masses, suggesting that they will collapse and fragment. The other clumps have virial masses comparable to their clump masses, suggesting that they are close to virial equilibrium with additional support from magnetic fields.

Clump "c8" is particularly interesting because it is not visible at Herschel/PACS 70 and $160 \mu \mathrm{m}$ bands and Spitzer/ MIPS $24 \mu \mathrm{m}$ band, indicating that it is very cold and maybe starless. The physical parameters (e.g., mass, density, size) of "c8" are similar to other Galactic massive starless clumps discovered in large surveys (e.g., Guzmán et al. 2015; Traficante et al. 2015; Contreras et al. 2017; Yuan et al. 2017). As noted earlier (Section 4.2.1), the magnetic field surrounding "c8" is pinched, hinting at gas inflow along the filament. The virial parameter $\left(\alpha_{\mathrm{vir}}\right)$ of "c 8 " is $\alpha_{\text {vir }}=M_{\text {vir }} / M_{\text {clump }} \leqslant 0.6$ even if we consider additional support from magnetic fields, suggesting that "c8" is undergoing gravitational collapse.

Figure 11 shows evidence of the collapse of "c8" from the asymmetric "blue-skewed profiles" of optically thick lines $\left(\mathrm{HCO}^{+}(1-0)\right.$ and $\mathrm{H}_{2} \mathrm{CO}\left(2_{1,2}-1_{1,1}\right)$ from KVN observations. The systemic velocity of "c 8 " is $44.9 \mathrm{~km} \mathrm{~s}^{-1}$, which is determined from Gaussian fitting to the single-peaked $\mathrm{H}^{13} \mathrm{CO}^{+}$ (1-0) line. In contrast, $\mathrm{HCO}^{+}(1-0)$ and $\mathrm{H}_{2} \mathrm{CO}\left(2_{1,2}-1_{1,1}\right)$ show double-peaked emission, with the blueshifted peak stronger than the redshifted one, and typical "blue-skewed profiles" for infall signature (Zhou et al. 1993). Such a "blue-skewed profile" of optically thick lines is commonly seen in surveys toward massive clumps (Wu \& Evans 2003; Fuller et al. 2005; Wu et al. 2007; Jin et al. 2016; Liu et al. 2016a), which can be interpreted as evidence for the global collapse of massive clumps (Liu et al. 2013a; Peretto et al. 2013). We highlight that, to our knowledge, "c8" could be the first discovered massive starless clump candidate exhibiting this characteristic infall profile.

We model the $\mathrm{HCO}^{+}(1-0)$ and $\mathrm{H}_{2} \mathrm{CO}\left(2_{1,2}-1_{1,1}\right)$ lines using RATRAN following Peretto et al. (2013) and Yuan et al. (2018). For the modeling, a power-law density profile $\left(\rho \propto r^{-1.5}\right)$ is assumed, and the kinetic temperature is set to be $13 \mathrm{~K}$. We have tried a grid of models by varying molecular abundances, infall velocities, and velocity dispersions. The resulting infall velocity inferred from the best models for $\mathrm{HCO}^{+}(1-0)$ is $0.32 \pm 0.04 \mathrm{~km} \mathrm{~s}^{-1}$, while the resulting infall velocity derived from the best models for $\mathrm{H}_{2} \mathrm{CO}\left(2_{1,2}-1_{1,1}\right)$ is $0.20 \pm 0.10 \mathrm{~km} \mathrm{~s}^{-1}$. Though the values are arguably the same within the uncertainties, the infall velocity traced by $\mathrm{H}_{2} \mathrm{CO}$ $\left(2_{1,2}-1_{1,1}\right)$ is smaller than that traced by $\mathrm{HCO}^{+}(1-0)$. Since the effective excitation density $\left(1.5 \times 10^{5} \mathrm{~cm}^{-1}\right)$ of $\mathrm{H}_{2} \mathrm{CO}\left(2_{1,2}\right.$ $\left.-1_{1,1}\right)$ at $10 \mathrm{~K}$ is much larger than that $\left(9.5 \times 10^{2} \mathrm{~cm}^{-1}\right)$ of $\mathrm{HCO}^{+}$(1-0) (Shirley 2015), $\mathrm{H}_{2} \mathrm{CO}\left(2_{1,2}-1_{1,1}\right)$ should trace denser, inner regions of the clump than $\mathrm{HCO}^{+}(1-0)$. Therefore, the gas inflow indicated by $\mathrm{H}_{2} \mathrm{CO}\left(2_{1,2}-1_{1,1}\right)$ and $\mathrm{HCO}^{+}(1-0)$ seems to be decelerated from the outer part to the inner part. The decelerated inflow may be caused by the enhanced magnetic field strength near the clump center, which will help resist gravity. Considering the uncertainties in the infall velocities, future work is needed.

Assuming a power-law density profile $\left(\rho \propto r^{-1.5}\right)$, the mass enclosed in $r_{o}$ is

$$
M=\int_{0}^{r_{o}} 4 \pi r^{2} \rho_{o}\left(\frac{r}{r_{o}}\right)^{-1.5} d r=\frac{4 \pi}{1.5} r_{o}^{3} \rho_{o},
$$

where $r_{o}$ is the outer radius and $\rho_{o}$ is the density at $r_{o}$. Therefore, the mass inflow rate at $r_{o}$ can be estimated using

$$
\dot{M}_{\text {in }}=4 \pi r_{o}^{2} \rho_{o} v_{\text {in }}=1.5 M v_{\text {in }} / r_{o} \text {. }
$$

We take the total clump mass $\left(\sim 200 M_{\odot}\right)$ for $M$ and clump radius $(0.28 \mathrm{pc})$ for $r_{o}$. We assume an infall velocity $v_{\text {in }}$ of $0.32 \mathrm{~km} \mathrm{~s}^{-1}$ obtained from the $\mathrm{HCO}^{+}(1-0)$ measurement because the mean volume density of "c 8 " is closer to the critical density of $\mathrm{HCO}^{+}(1-0)$. The inferred mass inflow rate $\left(\dot{M}_{\text {in }}\right)$ is thus $\sim 4 \times 10^{-4} M_{\odot} \mathrm{yr}^{-1}$. This mass inflow rate is consistent with those measured in other high-mass star-forming clumps (Wu et al. 2009, 2014; Sanhueza et al. 2010; Liu et al. 2011a, 2011b, 2013a, 2013b, 2016b; Ren et al. 2012; Peretto et al. 2013; Qin et al. 2016; Yuan et al. 2018). The clump mass of "c8" also exceeds the empirical threshold 
$\left(M>870 M_{\odot}(r / p c)^{1.33}\right)$ for high-mass star-forming clumps discovered by Kauffmann \& Pillai (2010). In addition, the clump mass of "c8" is also comparable to the masses of highmass starless clumps with similar radii cataloged by Yuan et al. (2017). All of these conditions indicate that "c8" has the potential to form high-mass stars.

The collapsing massive starless clump candidate "c8" may represent the very initial conditions for high-mass star formation and deserves more detailed studies at higher angular resolution. Indeed, searching for the existence or absence of high-mass prestellar cores, as has been done in other massive starless clump candidates (Beuther et al. 2013; Sanhueza et al. 2013, 2017; Tan et al. 2013; Liu et al. 2017; Contreras et al. 2018), is of great importance given that in "c8" the magnetic field and infall speed at large scales are now both known, unlike for the other studies.

\section{Summary}

We have studied the magnetic fields projected on the POS in the massive IRDC G035.39-00.33 from the JCMT/POL-2 polarization observations at $850 \mu \mathrm{m}$ and the large-scale kinematics from various molecular line observations. Our main findings are summarized below.

(1) From the deep JCMT/POL-2 observations, we identified a network of elongated structures covering a broad range of densities. The most massive filament $\left(F_{\mathrm{M}}\right)$ has a length of $\sim 6.8 \mathrm{pc}$, a mass of $\sim 2800 M_{\odot}$, and a line mass of $\sim 410 M_{\odot} \mathrm{pc}^{-1}$. The other fainter elongated structures have comparable lengths but are much less dense. A long elongated structure $\left(F_{\mathrm{W}}\right)$ having a length similar to $F_{\mathrm{M}}$ is connected to the northern end of $F_{\mathrm{M}} . F_{\mathrm{W}}$ is about four times less massive and less dense than $F_{\mathrm{M}}$.

(2) The orientations of the magnetic fields in the two less dense tails of $F_{\mathrm{M}}$ and some other less dense elongated structures (e.g., $F_{\mathrm{W}}$ ) tend to be parallel to the major axes of their respective skeletons. In contrast, magnetic fields in the densest regions of the middle part of $F_{\mathrm{M}}$ and some nodes at its junctions with other elongated structures $\left(F_{\mathrm{SW}}, F_{\mathrm{E}}\right.$, and $\left.F_{\mathrm{NE}}\right)$ are more perpendicular to the major axis.

(3) We claim that the massive filament $F_{\mathrm{M}}$ forms at the interface of two colliding clouds. The large-scale velocity gradient and multiple velocity components in $F_{\mathrm{M}}$ discovered in previous works can now be explained by the mixed gas distribution from these two colliding clouds. The northern end of $F_{\mathrm{M}}$ is more compressed by the cloud-cloud collision, and the magnetic fields therein are also compressed and aligned along the filament.

(4) $F_{M}$ is unstable against gravity if we only consider internal support from thermal pressure and turbulence. The magnetic field orientations suggest that the northern part of $F_{\mathrm{M}}$ may be dominated by a poloidal magnetic field component, which may provide additional support against gravity by increasing the effective critical mass per unit length. In contrast, the middle part of $F_{\mathrm{M}}$ may be dominated by a toroidal field component, which reduces the effective critical mass per unit length and makes the filament more unstable. The southern part of $F_{\mathrm{M}}$ is also unstable, even considering support from the magnetic field.
(5) Nine clumps with masses ranging from 16 to $219 M_{\odot}$ are identified along the main filament $F_{\mathrm{M}}$. The gravitational stability of the clumps is evaluated from a virial analysis considering internal support from thermal pressure, turbulence, and magnetic fields. Three clumps ("c1," "c2," and "c5") have virial masses much larger than their clump masses and hence are gravitationally unbound. The two most massive clumps ("c3" and "c8"), however, have clump masses larger than their virial masses even if the magnetic field support is considered, suggesting that they will collapse and fragment. The other clumps have virial masses comparable to their clump masses, suggesting that they are close to virial equilibrium, with additional support from magnetic fields.

(6) We discovered a massive $\left(\sim 200 M_{\odot}\right)$, collapsing starless clump candidate, "c8." This clump has a clump mass about two times larger than its virial mass, suggesting that it will collapse and fragment. The magnetic field surrounding "c8" is pinched, likely due to the accretion flow along its host filament. $\mathrm{HCO}^{+}(1-0)$ and $\mathrm{H}_{2} \mathrm{CO}\left(2_{1,2}\right.$ $-1_{1,1}$ ) spectra toward "c8" show a clear infall signature, i.e., the "blue-skewed profile." The infall velocities inferred from $\mathrm{HCO}^{+}(1-0)$ and $\mathrm{H}_{2} \mathrm{CO}\left(2_{1,2}-1_{1,1}\right)$ are $0.32 \pm 0.04 \mathrm{~km} \mathrm{~s}^{-1}$ and $0.20 \pm 0.10 \mathrm{~km} \mathrm{~s}^{-1}$, respectively. The mass inflow rate is $\sim 4 \times 10^{-4} M_{\odot} \mathrm{yr}^{-1}$. As this rate is consistent with those measured in other highmass star-forming clumps, "c8" likely has the potential ability to form high-mass stars. Higher-resolution (e.g., ALMA) data are needed to study the small-scale structure of this massive clump.

We thank the referee, Andrea Bracco, for very valuable comments and suggestions that have improved the content and clarity of this paper. Tie Liu is supported by EACOA fellowship. P.S.L. is supported by NASA ATP grant NNX13AB84G. M.J. acknowledges the support of the Academy of Finland grant no. 285769. J.M.a. acknowledges the support of ERC-2015-STG no. 679852 RADFEEDBACK. C.-P.Z. is supported by the National Natural Science Foundation of China 11703040. J.Y. is supported by the National Natural Science Foundation of China through grant 11503035. K.Q. acknowledges the support from National Natural Science Foundation of China (NSFC) through grants NSFC 11473011 and NSFC 11590781 . K.W. is supported by grant WA3628-1/1 of the German Research Foundation (DFG) through the priority program 1573 ("Physics of the Interstellar Medium"). C.W.L. was supported by Basic Science Research Program though the National Research Foundation of Korea (NRF) funded by the Ministry of Education, Science, and Technology (NRF2016R1A2B4012593). S.P.L. and K.M.P. acknowledge support from the Ministry of Science and Technology of Taiwan with grant MOST 106-2119-M-007-021-MY3. This research was partly supported by the OTKA grant NN-111016. This work was carried out in part at the Jet Propulsion Laboratory, which is operated for NASA by the California Institute of Technology. W.K. was supported by the Basic Science Research Program through the National Research Foundation of Korea (NRF2016R1C1B2013642). The James Clerk Maxwell Telescope is operated by the East Asian Observatory on behalf of The National Astronomical Observatory of Japan; Academia Sinica Institute of Astronomy and Astrophysics; the Korea Astronomy and Space Science Institute; the Operation, Maintenance and 


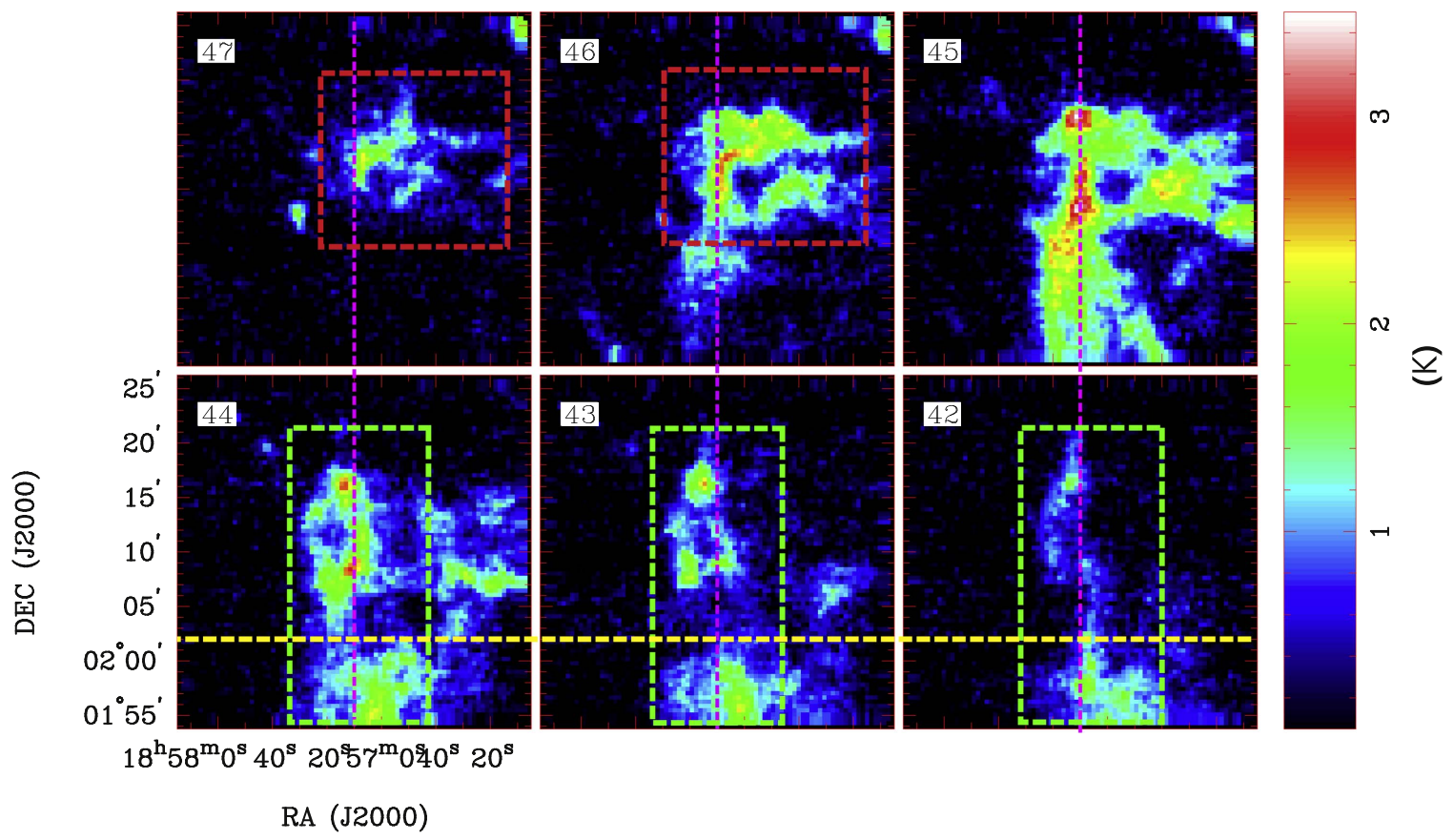

Figure 12. Channel maps of the ${ }^{13} \mathrm{CO}(1-0)$ line emission. The magenta vertical lines represent the long axis of the main filament. The red dashed boxes mark the cloud gas with redshifted velocity, while the green dashed boxes mark the cloud gas with blueshifted velocity. The yellow dashed line divides G035.39-main into two parts (southern and northern parts). The velocities of each panel are shown in the upper left corners.

Upgrading Fund for Astronomical Telescopes and Facility Instruments, budgeted from the Ministry of Finance (MOF) of China and administrated by the Chinese Academy of Sciences (CAS), as well as the National Key R\&D Program of China (No. 2017YFA0402700). Additional funding support is provided by the Science and Technology Facilities Council of the United Kingdom and participating universities in the United Kingdom and Canada. The KVN is a facility operated by the Korea Astronomy and Space Science Institute.

Software: Starlink software (Chapin et al. 2013; Jenness et al. 2013; Currie et al. 2014).

\section{Appendix \\ Channel Maps of ${ }^{13} \mathrm{CO}(1-0)$ Line Emission}

Figure 12 presents the channel maps of ${ }^{13} \mathrm{CO}(1-0)$ line emission for the $\sim 45 \mathrm{~km} \mathrm{~s}^{-1}$ component. From the channel maps, we identify two velocity-coherent clouds whose spatial distributions are distinctly different. The western cloud (hereafter denoted as G035.39-west) with redshifted velocity is mainly distributed in the northwest part of the images, as marked by the red dashed boxes in the 46 and $47 \mathrm{~km} \mathrm{~s}^{-1}$ channel maps. The cloud with blueshifted velocity is a long $(\sim 20 \mathrm{pc})$ filamentary cloud (hereafter denoted as G035.39-main) distributed along the north-south direction, as marked by the green dashed boxes in the 42, 43, and $44 \mathrm{~km} \mathrm{~s}^{-1}$ channel maps. The G035.39-main cloud is divided into two parts by the yellow dashed line in the channel maps. The two parts are connected in velocity space. The high-velocity emissions of G035.39-west and G035.39-main clouds are well separated by the magenta dashed line in the channel maps, which marks the major axis of the massive filament $F_{\mathrm{M}}$. The line emission of G035.39-west at its highvelocity $\left(47 \mathrm{~km} \mathrm{~s}^{-1}\right)$ channel is mainly distributed to the west of $F_{\mathrm{M}}$. On the other hand, the high-velocity $\left(43-44 \mathrm{~km} \mathrm{~s}^{-1}\right)$ emission of the northern part of G035.39-main is mainly distributed to the east of the $F_{\mathrm{M}}$. The brightest ${ }^{13} \mathrm{CO}(1-0)$ line emission is in the $\sim 45 \mathrm{~km} \mathrm{~s}^{-1}$ channel, where two clouds overlap.

\section{ORCID iDs}

Tie Liu (i) https://orcid.org/0000-0002-5286-2564 Pak Shing Li (1) https://orcid.org/0000-0001-8077-7095 Kee-Tae Kim (1) https://orcid.org/0000-0003-2412-7092 Sheng-Yuan Liu (10) https://orcid.org/0000-0003-4603-7119 Jinghua Yuan (1) https://orcid.org/0000-0001-8060-3538 Ken'ichi Tatematsu (i) https://orcid.org/0000-0002-8149-8546 Qizhou Zhang (1) https://orcid.org/0000-0003-2384-6589 Derek Ward-Thompson (ib https://orcid.org/0000-00031140-2761

Paul F. Goldsmith @i https://orcid.org/0000-0002-6622-8396 P. M. Koch (1) https://orcid.org/0000-0003-2777-5861 Patricio Sanhueza (i) https://orcid.org/0000-0002-7125-7685 Sung-ju Kang (1) https://orcid.org/0000-0002-5004-7216 Huei-Ru Chen (1) https://orcid.org/0000-0002-9774-1846 Yuefang Wu (1) https://orcid.org/0000-0002-5076-7520 Vlas Sokolov (1) https://orcid.org/0000-0002-5327-4289 Glenn J. White (1) https://orcid.org/0000-0002-7126-691X Ke Wang (i) https://orcid.org/0000-0002-7237-3856 Kate M Pattle (1) https://orcid.org/0000-0002-8557-3582 Archana Soam (ㄷ https://orcid.org/0000-0002-6386-2906 Shih-Ping Lai (1) https://orcid.org/0000-0001-5522-486X Geumsook Park (1) https://orcid.org/0000-0001-8467-3736 Keping Qiu (ib https://orcid.org/0000-0002-5093-5088 Chuan-Peng Zhang (i) https://orcid.org/0000-0002-4428-3183 Chakali Eswaraiah (1) https://orcid.org/0000-0003-4761-6139 Jane Greaves (i) https://orcid.org/0000-0002-3133-413X Woojin Kwon (1) https://orcid.org/0000-0003-4022-4132 Hua-bai Li (i) https://orcid.org/0000-0003-2641-9240 Harriet Parsons (10 https://orcid.org/0000-0002-6327-3423 
Mengyao Tang (1) https://orcid.org/0000-0001-9160-2944

L. V. Toth (1) https://orcid.org/0000-0002-5310-4212 Jan Wouterloot (1) https://orcid.org/0000-0002-4694-6905

\section{References}

Alina, D., Ristorcelli, I., Montier, L., et al. 2017, arXiv:1712.09325 André, P., Di Francesco, J., Ward-Thompson, D., et al. 2014, in Protostars and Planets VI, ed. H. Beuther et al. (Tucson: Univ. Arizona Press), 27 Auddy, S., Basu, S., Kudoh, T., et al. 2016, ApJ, 831, 46

Barnes, A. T., Kong, S., Tan, J. C., et al. 2016, MNRAS, 458, 1990 Berry, D. S. 2015, A\&C, 10, 22

Berry, D. S., Reinhold, K., Jenness, T., \& Economou, F. 2007, in ASP Conf. Ser. 376, Astronomical Data Analysis Software and Systems XVI, ed. R. A. Shaw, F. Hill, \& D. J. Bell (San Francisco, CA: ASP), 425 Bertoldi, F., \& McKee, C. F. 1992, ApJ, 395, 140

Beuther, H., Linz, H., Tackenberg, J., et al. 2013, A\&A, 553, A115

Bisbas, T. G., Tan, J. C., Csengeri, T., et al. 2018, MNRAS, in press (arXiv:1803.00566)

Chandrasekhar, S., \& Fermi, E. 1953, ApJ, 118, 116

Chapin, E. L., Berry, D. S., Gibb, A. G., et al. 2013, MNRAS, 430, 2545

Chapman, N. L., Goldsmith, P. F., Pineda, J. L., et al. 2011, ApJ, 741, 21

Contreras, Y., Garay, G., Rathborne, J. M., et al. 2016, MNRAS, 456, 2041

Contreras, Y., Rathborne, J. M., Guzman, A., et al. 2017, MNRAS, 466, 340

Contreras, Y., Sanhueza, P., Jackson, J. M., et al. 2018, ApJ, in press (arXiv:1805.01802)

Cox, N. L. J., Arzoumanian, D., André, Ph., et al. 2016, A\&A, 590, 110

Crutcher, R. M., Nutter, D. J., Ward-Thompson, D., \& Kirk, J. M. 2004, ApJ, 600, 279

Crutcher, R. M., Wandelt, B., Heiles, C., et al. 2010, ApJ, 725, 466

Currie, M. J., Berry, D. S., Jenness, T., et al. 2014, in ASP Conf. Ser. 485, Astronomical Data Analysis Software and Systems XXIII, ed. N. Manset \& P. Forshay (San Francisco, CA: ASP), 391

Davis, L. J., \& Greenstein, J. L. 1951, ApJ, 114, 206

Eden, D. J., Moore, T. J. T., Plume, R., et al. 2017, MNRAS, 469, 2163

Federrath, C. 2016, MNRAS, 457, 375

Fiege, J. D., \& Pudritz, R. E. 2000, MNRAS, 311, 85

Friberg, P., Bastien, P., Berry, D., et al. 2016, Proc. SPIE, 9914, 991403

Fuller, G. A., Williams, S. J., \& Sridharan, T. K. 2005, A\&A, 442, 949

Giannetti, A., Wyrowski, F., Brand, J., et al. 2014, A\&A, 570, 65

Girart, J. M., Frau, P., Zhang, Q., et al. 2013, ApJ, 772, 69

Gomez, G. C., Vazquez-Semadeni, E., \& Zamora-Aviles, M. 2018, MNRAS, submitted (arXiv:1801.03169)

Guzmán, A. E., Sanhueza, P., Contreras, Y., et al. 2015, ApJ, 815, 130

Hacar, A., Kainulainen, J., Tafalla, M., Beuther, H., \& Alves, J. 2016, A\&A, 587, 97

Hacar, A., Tafalla, M., Kauffmann, J., \& Kovács, A. 2013, A\&A, 554, 55

Heitsch, F., Zweibel, E. G., Mac Low, M.-M., Li, P., \& Norman, M. L. 2001, ApJ, 561, 800

Henshaw, J. D., Caselli, P., Fontani, F., et al. 2013, MNRAS, 428, 3425

Henshaw, J. D., Caselli, P., Fontani, F., et al. 2016, MNRAS, 463, 146

Henshaw, J. D., Caselli, P., Fontani, F., Jiménez-Serra, I., \& Tan, J. C. 2014, MNRAS, 440, 2860

Henshaw, J. D., Jiménez-Serra, I., Longmore, S. N., et al. 2017, MNRAS, 464,31

Hernandez, A. K., \& Tan, J. C. 2015, ApJ, 809, 154

Holland, W. S., Bintley, D., Chapin, E. L., et al. 2013, MNRAS, 430, 2513 Inoue, T., \& Fukui, Y. 2013, ApJ, 774L, L31

Jackson, J. M., Finn, S. C., Chambers, E. T., et al. 2010, ApJ, 791L, 185

Jenness, T., Chapin, E. L., Berry, D. S., et al. 2013, SMURF: SubMillimeter

User Reduction Facility, Astrophysics Source Code Library, ascl:1310.007

Jiménez-Serra, I., Caselli, P., Fontani, F., et al. 2014, MNRAS, 439, 1996

Jiménez-Serra, I., Caselli, P., Tan, J. C., et al. 2010, MNRAS, 406, 187

Jin, M., Lee, J.-E., Kim, K.-T., et al. 2016, ApJS, 225, 21

Juvela, M., Guillet, V., Liu, T., et al. 2018b, A\&A, submitted

Juvela, M., He, J., Pattle, K., et al. 2018a, A\&A, 612, A71

Juvela, M., Ristorcelli, I., Montier, L. A., et al. 2010, A\&A, 518, L93

Juvela, M., Ristorcelli, I., Pagani, L., et al. 2012, A\&A, 541, 12

Kainulainen, J., \& Tan, J. C. 2013, A\&A, 549, 53

Kauffmann, J., \& Pillai, T. 2010, ApJL, 723, L7

Kim, J., Lee, J.-E., Liu, T., et al. 2017, ApJS, 231, 9

Kim, K.-T., Byun, D.-Y., Je, D.-H., et al. 2011, JKAS, 44, 81

Klassen, M., Pudritz, R. E., \& Kirk, H. 2017, MNRAS, 465, 2254

Koch, E. W., \& Rosolowsky, E. W. 2015, MNRAS, 452, 3435
Koch, P. M., Tang, Y.-W., \& Ho, P. T. P. 2012, ApJ, 747, 79

Koch, P. M., Tang, Y.-W., Ho, P. T. P., et al. 2014, ApJ, 797, 99

Kwon, J., Doi, Y., Tamura, M., et al. 2018, ApJ, 859, 4

Li, H.-B., Dowell, C. D., Goodman, A., Hildebrand, R., \& Novak, G. 2009, ApJ, 704, 891

Li, H.-B., Yuen, K. H., Otto, F., et al. 2015a, Natur, 520, 518

Li, P. S., Klein, R. I., \& McKee, C. F. 2017, MNRAS, 473, 4220

Li, P. S., McKee, C. F., \& Klein, R. I. 2015b, MNRAS, 452, 2500

Liu, T., Kim, K.-T., Juvela, M., et al. 2018, ApJS, 234, 28

Liu, T., Kim, K.-T., Yoo, H., et al. 2016a, ApJ, 829, 59

Liu, T., Lacy, J., Li, P. S., et al. 2017, ApJ, 849, 25

Liu, T., Wu, Y., Liu, S.-Y., et al. 2011a, ApJ, 730, 102

Liu, T., Wu, Y., Mardones, D., et al. 2015, PKAS, 30, 79

Liu, T., Wu, Y., Wu, J., et al. 2013a, MNRAS, 436, 1335

Liu, T., Wu, Y., \& Zhang, H. 2012, ApJS, 202, 4

Liu, T., Wu, Y., \& Zhang, H. 2013b, ApJ, 776, 29

Liu, T., Wu, Y., \& Zhang, H. 2013c, ApJ, 775L, 2L

Liu, T., Wu, Y., Zhang, Q., et al. 2011b, ApJ, 728, 91

Liu, T., Zhang, Q., Kim, K.-T., et al. 2016b, ApJ, 824, 31

Liu, T., Zhang, Q., Kim, K.-T., et al. 2016c, ApJS, 222, 7

Lu, X., Zhang, Q., Liu, H. B., et al. 2018, ApJ, 855, 9

Malinen, J., Montier, L., Montillaud, J., et al. 2016, MNRAS, 460, 1934

Meng, F., Wu, Y., Liu, T., et al. 2013, ApJS, 209, 37

Molinari, S., Swinyard, B., Bally, J., et al. 2010, A\&A, 518, L100

Montillaud, J., Juvela, M., \& Rivera-Ingraham, A. 2015, A\&A, 584, A92

Moore, T. J. T., Plume, R., Thompson, M. A., et al. 2015, MNRAS, 453, 4264

Nguyen Luong, Q., Motte, F., Hennemann, M., et al. 2011, A\&A, 535, 76

Ostriker, E. C., Stone, J. M., \& Gammie, C. F. 2001, ApJ, 546, 980

Ostriker, J. 1964, ApJ, 140, 1056

Palmeirim, P., André, Ph., Kirk, J., et al. 2013, A\&A, 550, 38

Panopoulou, G. V., Psaradaki, I., Skalidis, R., et al. 2017, MNRAS, 466, 2529

Pattle, K., Ward-Thompson, D., Berry, D., et al. 2017, ApJ, 846, 122

Peretto, N., Fuller, G. A., Duarte-Cabral, A., et al. 2013, A\&A, 555, 112

Pillai, T., Kauffmann, J., Tan, J. C., et al. 2015, ApJ, 799, 74

Pillai, T., Kauffmann, J., Wyrowski, F., et al. 2011, A\&A, 530, A118

Planck Collaboration, Ade, P. A. R., Aghanim, N., et al. 2011a, A\&A, 536, 23

Planck Collaboration, Ade, P. A. R., Aghanim, N., et al. 2011b, A\&A, 536, 22

Planck Collaboration, Ade, P. A. R., Aghanim, N., et al. 2016a, A\&A, 586, 138

Planck Collaboration, Adam, R., Ade, P. A. R., et al. 2016b, A\&A, 586, 135

Planck Collaboration, Ade, P. A. R., Aghanim, N., et al. 2016c, A\&A, 594, 28

Qin, S.-L., Schilke, P., Wu, J., et al. 2016, MNRAS, 456, 2681

Qiu, K., Zhang, Q., Menten, K. M., et al. 2013, ApJ, 779, 182

Qiu, K., Zhang, Q., Menten, K. M., et al. 2014, ApJ, 794L, 18

Ren, Z., Wu, Y., Zhu, M., et al. 2012, MNRAS, 422, 1098

Rivera-Ingraham, A., Ristorcelli, I., Juvela, M., et al. 2016, A\&A, 591, A90

Rivera-Ingraham, A., Ristorcelli, I., Juvela, M., et al. 2017, A\&A, 601, A94

Sanhueza, P., Garay, G., Bronfman, L., et al. 2010, ApJ, 715, 18

Sanhueza, P., Jackson, J. M., Foster, J. B., et al. 2012, ApJ, 756, 60

Sanhueza, P., Jackson, J. M., Foster, J. B., et al. 2013, ApJ, 773, 123

Sanhueza, P., Jackson, J. M., Zhang, Q., et al. 2017, ApJ, 841, 97

Santos, F. P., Busquet, G., Franco, G. A. P., et al. 2016, ApJ, 832, 186

Shirley, Y. L. 2015, PASP, 127, 299

Simon, R., Rathborne, J. M., Shah, R. Y., Jackson, J. M., \& Chambers, E. T. 2006, ApJ, 653, 1325

Soam, A., Pattle, K., Ward-Thompson, D., et al. 2018, ApJ, in press (arXiv:1805.06131)

Sokolov, V., Wang, K., Pineda, J. E., et al. 2017, A\&A, 606, 133

Soler, J. D., Ade, P. A. R., Angilè, F. E., et al. 2017, A\&A, 603, 64

Soler, J. D., \& Hennebelle, P. 2017, A\&A, 607, 2

Tan, J. C., Kong, S., Butler, M. J., Caselli, P., \& Fontani, F. 2013, ApJ, 779, 96 Tang, M., Liu, T., Qin, S.-L., et al. 2018a, ApJ, 856, 141

Tang, Y.-W., Koch, P. M., Peretto, N., et al. 2018b, ApJ, submitted

Tatematsu, K., Liu, T., Ohashi, S., et al. 2017, ApJS, 228, 12

Traficante, A., Fuller, G. A., Peretto, N., Pineda, J. E., \& Molinari, S. 2015, MNRAS, 451, 3089

Van der Tak, F. F. S., Black, J. H., Schöier, F. L., Jansen, D. J., \& van Dishoeck, E. F. 2007, A\&A, 468, 627

Wang, K., Testi, L., Burkert, A., et al. 2016, ApJS, 226, 9

Ward-Thompson, D., Pattle, K., Bastien, P., et al. 2017, ApJ, 842, 66

Wu, B., Tan, J. C., Nakamura, F., et al. 2017, ApJ, 835, 137

Wu, B., Van Loo, S., Tan, J. C., et al. 2015, ApJ, 811, 56

Wu, J., \& Evans, N. J., II 2003, ApJ, 592L, 79

Wu, Y., Henkel, C., Xue, R., et al. 2007, ApJ, 669L, 37 
Wu, Y., Liu, T., Meng, F., et al. 2012, ApJ, 756, 76

Wu, Y., Liu, T., \& Qin, S.-L. 2014, ApJ, 791, 123

Wu, Y., Qin, S.-L., Guan, X., et al. 2009, ApJ, 697L, 116

Yi, H.-W., Lee, J.-E., Liu, T., et al. 2018, ApJS, in press (arXiv:1805.05738)

Yuan, J., Li, J.-Z., Wu, Y., et al. 2018, ApJ, 852, 12

Yuan, J., Wu, Y., Ellingsen, S. P., et al. 2017, ApJS, 231, 11
Yuan, J., Wu, Y., Liu, T., et al. 2016, ApJ, 820, 37

Zhang, C.-P., Liu, T., Yuan, J., et al. 2018, ApJS, in press (arXiv:1805.03883)

Zhang, C.-P., Yuan, J.-H., Li, G.-X., et al. 2018, A\&A, 598, A76

Zhang, Q., Qiu, K., Girart, J. M., et al. 2014, ApJ, 792, 116

Zhang, T., Wu, Y., Liu, T., et al. 2016, ApJS, 224, 43

Zhou, S., Evans, N. J., II, Koempe, C., et al. 1993, ApJ, 404, 232 